Article

\title{
Deep Residual CNN-Based Ocular Recognition Based on Rough Pupil Detection in the Images by NIR Camera Sensor
}

\author{
Young Won Lee, Ki Wan Kim, Toan Minh Hoang, Muhammad Arsalan and Kang Ryoung Park* \\ Division of Electronics and Electrical Engineering, Dongguk University, 30 Pildong-ro 1-gil, Jung-gu, \\ Seoul 04620, Korea; lyw941021@dongguk.edu (Y.W.L.); yawara18@dongguk.edu (K.W.K.); \\ hoangminhtoant211@gmail.com (T.M.H.); arsal@dongguk.edu (M.A.) \\ * Correspondence: parkgr@dongguk.edu; Tel.: +82-10-3111-7022; Fax: +82-2-2277-8735
}

Received: 21 December 2018; Accepted: 15 February 2019; Published: 18 February 2019

\begin{abstract}
Accurate segmentation of the iris area in input images has a significant effect on the accuracy of iris recognition and is a very important preprocessing step in the overall iris recognition process. In previous studies on iris recognition, however, the accuracy of iris segmentation was reduced when the images of captured irises were of low quality due to problems such as optical and motion blurring, thick eyelashes, and light reflected from eyeglasses. Deep learning-based iris segmentation has been proposed to improve accuracy, but its disadvantage is that it requires a long processing time. To resolve this problem, this study proposes a new method that quickly finds a rough iris box area without accurately segmenting the iris region in the input images and performs ocular recognition based on this. To address this problem of reduced accuracy, the recognition is performed using the ocular area, which is a little larger than the iris area, and a deep residual network (ResNet) is used to resolve the problem of reduced recognition rates due to misalignment between the enrolled and recognition iris images. Experiments were performed using three databases: Institute of Automation Chinese Academy of Sciences (CASIA)-Iris-Distance, CASIA-Iris-Lamp, and CASIA-Iris-Thousand. They confirmed that the method proposed in this study had a higher recognition accuracy than existing methods.
\end{abstract}

Keywords: biometrics; rough pupil detection; ocular recognition; deep ResNet

\section{Introduction}

Due to recent developments in technology, iris recognition is being used for personal authentication in smartphones [1]. The basic concept of iris recognition that is used conventionally was first proposed in a patent by Flom et al. [2], and the fundamental basis for most iris recognition systems that are currently used was created by Daugman et al. [3-6]. Generally, iris recognition performance is influenced by the environment in which the image is captured (e.g., a noisy environment, low resolution, optical or motion blurring, specular reflection and in-plane rotation, off-angle, off-axis). To prevent such environmental influences, iris images were captured in a constrained environment. Furthermore, there were cases in which iris recognition with a visible camera was difficult in Asian people due to the dark color of their irises, which is caused by a high level of the pigment melanin. If the images were captured by a near-infrared (NIR) light camera sensor, full iris features could be obtained despite a dark iris color [7-12]. However, an NIR camera requires an NIR illuminator, and using the latter increases the size and price of the system. Therefore, studies are still being conducted on iris recognition using images captured by visible camera sensors, which are relatively easy to set up [13-16]. However, there are cases in which more noise can occur when a visible camera is used, 
resulting in a reduced recognition performance. Problems such as optical or motion blur, specular reflection, and in-plane rotation can occur when iris images are captured with both NIR and visible light cameras. If this occurs, it becomes difficult to accurately segment the iris area, which could have a significant effect on the accuracy of iris recognition. Segmenting the iris area is also a very important preprocessing step in the overall iris recognition process. The problems that occur in a constrained image acquisition environment become even more severe in an unconstrained environment. To resolve these problems, more acquisition system designed in a more complex manner are required, along with high quality images and processing performances $[7,8,13,14]$.

There are studies which resolve those problems by using an ocular area that is wider than the conventional iris area [17]. The ocular area can be used in applications in which a confirmation of identity is performed through the surrounding skin when an image of the iris cannot be captured reliably. Furthermore, if the image is captured from a long distance, its resolution is low and the recognition performance is reduced because of this. Using the ocular area has the advantage of improving performance in such cases; however, it is still difficult to extract the features that are important for personal recognition from ocular areas outside the iris, including skin with little texture. In addition, there are still problems with reduced recognition rates due to misalignment between the enrolled and recognition ocular images. To consider these issues, we propose deep learning-based ocular recognition based on rough pupil detection.

This paper is organized as follows: Section 2 compares the advantages and disadvantages of existing iris and ocular recognition methods. Section 3 describes the contributions of this study and Section 4 shows ocular recognition using the deep residual network (ResNet) proposed in this study. Section 5 presents the experimental results and analysis, and Section 6 presents the conclusions of the study.

\section{Related Works}

In iris recognition, the iris segmentation performance and texture quality in the captured image are greatly affected by the image capturing environment. Thus, this is one aspect that makes it difficult to create an iris recognition system for a variety of environments. To resolve this, the development of recognition methods that use the ocular region, which is somewhat larger than the iris region, has been attempted [17-25]. Rattani et al. [19] introduced a variety of studies on existing ocular recognition methods. In order to extract features from a visible input image using the ocular region, Park et al. [17] used a Hough transformation to detect the iris area and performed interest point detection and region sampling based on the detected image. After image alignment, global descriptors and features were extracted, and then a scale invariant feature transform (SIFT) was performed using gradient orientation (GO) and local binary pattern (LBP) as local descriptors in order to extract local features and perform matching [17]. Cho et al. [20] proposed a method that can improve recognition accuracy through adaptive bit shifting and matching after defining circular regions of interest (ROI) for creating a periocular area based on the iris radius and then converting this area to polar coordinates to make it robust against eye rotation. Rodriguez et al. [21] studied ocular recognition using a quadratic correlation filter. Ross et al. [18] studied ocular recognition through matching score fusion using various feature extraction techniques, such as gradient orientation histograms $(\mathrm{GOH})$, modified SIFT (m-SIFT), and probabilistic deformation models (PDM).

In addition to this, they studied methods that can perform fusion and recognition to achieve the advantages of both iris and ocular recognition [22-26]. Boddeti et al. [22] studied matching score-level fusion of the iris and ocular regions through fusion optimal trade-off synthetic discriminant function (OTSDF) correlation filtering, and maximum a posterior probability (MAP) estimation in order to perform recognition on challenging ocular images captured in an NIR camera sensor environment. Verma et al. [23] performed periocular recognition on ocular images captured in a long-distance NIR camera sensor environment using traditional iris recognition, a pyramid histogram of oriented gradients (PHOG), and gist methods and then performed fusion and classification using a random 
decision forest (RDF). Oishi et al. [24] performed a study on the fusion of both iris and periocular recognition in a mobile device using adaptive boosting (Adaboost). Tan et al. [25] proposed a method of score fusion-based iris and periocular recognition using images captured by a visible light camera sensor in an uncooperative environment. Lee et al. [26] proposed a recognition method that performs a fusion on the results by inputting three different areas including the existing segmented iris area and a wider area around it into three convolutional neural network (CNN) models. Moreover, Ahuja et al. [27] proposed a recognition method that fused the recognition results of the iris area, the ocular area, and an area slightly larger than the ocular area. Processing of the ocular region was performed using features learned through the VisobNet CNN. Processing of the iris region was performed using features extracted through prior segmentation processing and root-SIFT. Processing of the face was performed using the OpenFace face recognition method [28]. However, the methods in these existing studies have issues in that recognition performance is affected by ocular region detection accuracy and the recognition accuracy is worse than iris segmentation-based iris recognition.

In previous research [29], a super-resolution reconstruction method based on $\mathrm{CNN}$ for iris recognition was proposed. In [30], the authors proposed periocular recognition by explicit attention to critical regions in deep CNN. Drozdowski et al. proposed the method of detection of glasses in ocular images [31]. In previous research [32], a classification method of heterogeneous objects including ocular images was proposed. Reddy et al. proposed the multi-frame super resolution for ocular recognition [33]. Although it is not ocular recognition but face recognition, Ahonen et al. proposed LBP-based face recognition with its application, and their method showed the promising recognition accuracies [34].

To address the problems of previous researches, this study proposes an ocular recognition method that quickly selects a rough iris box area without accurate iris segmentation. To address the problem of the reduced accuracy of recognition based on the rough selection, an ocular area somewhat larger than the iris area is used to perform recognition, and a deep ResNet is used to resolve the problem of a reduced recognition rate caused by misalignment between the enrolled and recognition images.

Table 1 is a summarized comparison of the method proposed in this study and existing methods. In Table 1, "A"-“"N" means "CASIA-Iris-Distance database", "NICE.II training database", "face and ocular challenge series (FOCS) database", "face recognition grand challenge (FRGC) database", "mobile iris challenge evaluation (MICHE) database", "CASIA-Iris-Lamp database", "CASIA-Iris-Thousand database", "self-collected dataset database", "Q-FIRE iris dataset", "CASIA cross sensor iris dataset", “ND-iris-0405", “ND-CrossSensor-Iris-2013”, “MBGC NIR v2 database”, and "VISOB dataset", respectively. 
Table 1. Comparisons of proposed and previous researches ( $\mathrm{d}^{\prime}$, EER, GAR, and FAR mean d-prime value, equal error rate, genuine acceptance rate, and false acceptance rate, respectively. Their concepts are explained in Sections 4.4 and 5.3).

\begin{tabular}{|c|c|c|c|c|c|c|}
\hline $\begin{array}{c}\text { Iris } \\
\text { Segmentation }\end{array}$ & $\begin{array}{l}\text { Periocular } \\
\text { Region }\end{array}$ & Using Feature & Method & Accuracy & Advantage & Disadvantage \\
\hline \multirow{7}{*}{ Included } & \multirow[b]{2}{*}{ Not used } & \multirow{5}{*}{$\begin{array}{l}\text { Hand-crafted } \\
\quad \text { feature }\end{array}$} & Log Gabor filter, LDA, and BSA [35] & EER of $3.12 \sim 3.465 \%$ (A) & \multirow{5}{*}{$\begin{array}{l}\text { Captured iris images are less } \\
\text { affected by external light than those } \\
\text { from a visible light camera }\end{array}$} & \multirow{5}{*}{$\begin{array}{l}\text { Equipment becomes large-sized and } \\
\text { expensive due to use of NIR camera and } \\
\text { NIR lighting }\end{array}$} \\
\hline & & & $\begin{array}{l}\text { Pre-classification and in-plane rotation } \\
\text { angle estimation for both eyes, then } \\
\text { matching via bit-shifting [8] }\end{array}$ & EER of $4.3006 \%(A)$ & & \\
\hline & \multirow{10}{*}{ Used } & & $\begin{array}{l}\text { Fusion-OTSDF, correlation filtering, and } \\
\text { MAP estimation [22] }\end{array}$ & EER of $23.83 \sim 26.81 \%$ (C) & & \\
\hline & & & $\begin{array}{l}\text { Fusion and classification with RDF } \\
\text { using PHOG and gist method [23] }\end{array}$ & $\begin{array}{l}\text { GAR of } 61.5 \% \text { at } 0.1 \% \text { FAR (A) } \\
\text { GAR of } 21 \% \text { at } 1 \% \text { FAR (C) }\end{array}$ & & \\
\hline & & & Adaboost and OSIRIS [24] & EER of $3.6 \sim 3.9 \%(\mathrm{~A})$ & & \\
\hline & & \multirow{2}{*}{ Deep feature } & Using three CNNs [26] & $\begin{array}{l}\text { EER of } 3.04 \sim 3.08 \% \text { (A) } \\
\quad \text { EER of } 10.36 \% \text { (B) } \\
\text { EER of } 16.25 \sim 17.9 \% \text { (E) }\end{array}$ & \multirow{2}{*}{$\begin{array}{l}\text { By using visible light camera } \\
\text { without NIR lighting, equipment is } \\
\text { small-sized and low cost, has no } \\
\text { reflected light due to NIR lighting }\end{array}$} & \multirow{2}{*}{$\begin{array}{l}\text { - Iris quality is slightly lower than a normal } \\
\text { NIR environment image due to nearby } \\
\text { lighting noise and ghost effects } \\
\text { - Difficult to capture iris patterns in visible } \\
\text { light for races with little melanin pigment }\end{array}$} \\
\hline & & & $\begin{array}{l}\text { Fusion of the recognitions by iris, ocular, } \\
\text { and the area larger than ocular [27] }\end{array}$ & EER of $5.7 \%(E)$ & & \\
\hline \multirow{5}{*}{ Not included } & & \multirow{5}{*}{$\begin{array}{l}\text { Hand-crafted } \\
\text { feature }\end{array}$} & $\begin{array}{l}\text { GOH, PDM, m-SIFT, and fusion by } \\
\text { weighted SUM rule [18] }\end{array}$ & EER of $18.8 \%(C)$ & \multirow{3}{*}{$\begin{array}{l}\text { Captured iris images are less } \\
\text { affected by external light than those } \\
\text { from a visible light camera }\end{array}$} & \multirow{3}{*}{$\begin{array}{l}\text { Equipment becomes large-sized and } \\
\text { expensive due to use of NIR camera and } \\
\text { NIR lighting }\end{array}$} \\
\hline & & & Quadratic correlation filter [21] & $\begin{array}{l}\text { Classification accuracy of } 75 \% \\
\text { (H) }\end{array}$ & & \\
\hline & & & $\begin{array}{l}\text { Conversion of periocular region into } \\
\text { polar-coordinates and matching via LBP } \\
\text { and adaptive bit shifting [20] }\end{array}$ & EER of $10.0172 \%(\mathrm{~A})$ & & \\
\hline & & & Multi-order statistical descriptors [32] & $\begin{array}{l}\text { Classification accuracy of } \\
93.33 \%(\mathrm{M})\end{array}$ & Intensive training is not required & $\begin{array}{l}\text { Performance enhancement is limited by } \\
\text { using hand-crafted feature }\end{array}$ \\
\hline & & & $\begin{array}{l}\text { Multi-frame super resolution } \\
\text { reconstruction based on deblurring by } \\
\text { CNN [33] }\end{array}$ & EER of $19.54 \sim 28.06 \%(\mathrm{~N})$ & $\begin{array}{l}\text { Image resolution and blurring of } \\
\text { ocular image can be enhanced }\end{array}$ & $\begin{array}{l}\text { Using hand-crafted feature and } \\
\text { unsophisticated matcher produces low } \\
\text { recognition accuracy }\end{array}$ \\
\hline
\end{tabular}


Table 1. Cont.

\begin{tabular}{|c|c|c|c|c|c|c|}
\hline $\begin{array}{c}\text { Iris } \\
\text { Segmentation }\end{array}$ & $\begin{array}{l}\text { Periocular } \\
\text { Region }\end{array}$ & Using Feature & Method & Accuracy & Advantage & Disadvantage \\
\hline \multirow{3}{*}{ Included } & \multirow{3}{*}{ Not used } & \multirow{4}{*}{ Deep feature } & $\begin{array}{l}\text { Iris recognition with off-the-shelf CNN } \\
\text { features [36] }\end{array}$ & $\begin{array}{c}\text { Recognition accuracy of } \\
98.8 \%(\mathrm{G}) \\
\text { Recognition accuracy of } \\
98.7 \%(\mathrm{H})\end{array}$ & High recognition accuracy & $\begin{array}{l}\text { - Performance is affected by the accurate iris } \\
\text { segmentation } \\
\text { - The complexity of DenseNet-201 is large, } \\
\text { which requires huge training time. }\end{array}$ \\
\hline & & & $\begin{array}{l}\text { DeepIris based on pairwise filter } \\
\text { bank [37] }\end{array}$ & $\begin{array}{l}\text { EER of } 0.15 \%(\mathrm{I}) \\
\text { EER of } 0.31 \%(\mathrm{~J})\end{array}$ & $\begin{array}{l}\text { Measure the accuracies according to } \\
\text { various capturing distances and } \\
\text { types of camera sensors }\end{array}$ & $\begin{array}{l}\text { - Performance is affected by the accurate iris } \\
\text { segmentation } \\
\text { - Performance enhancement is limited by } \\
\text { using shallow CNN }\end{array}$ \\
\hline & & & DeepIrisNet [38] & $\begin{array}{c}\text { EER of } 2.19 \%(\mathrm{~K}) \\
\text { EER of } 1.82 \sim 2.4 \%(\mathrm{~L})\end{array}$ & $\begin{array}{l}\text { Compare the accuracies according to } \\
\text { various types of segmentation } \\
\text { method, in-plane rotations, input } \\
\text { size, train size, and network size. }\end{array}$ & $\begin{array}{l}\text { - Performance is affected by the accurate iris } \\
\text { segmentation } \\
\text { - Performance enhancement is limited by } \\
\text { using shallow CNN }\end{array}$ \\
\hline Not included & Used & & $\begin{array}{l}\text { Deep ResNet-based recognition } \\
\text { (Proposed method) }\end{array}$ & $\begin{array}{l}\text { EER of } 2.1625 \%(\mathrm{~A}) \\
\text { EER of } 1.595 \%(\mathrm{~F}) \\
\text { EER of } 1.331 \%(\mathrm{G})\end{array}$ & $\begin{array}{l}\text { Not require iris segmentation while } \\
\text { maintaining the accuracy by deep } \\
\text { ResNet }\end{array}$ & $\begin{array}{l}\text { Require the procedure of intensive training } \\
\text { of ResNet }\end{array}$ \\
\hline
\end{tabular}




\section{Contributions}

Our research is novel in the following four ways compared to previous works:

- We propose a NIR camera-based ocular recognition method using deep features without iris segmentation, as shown in Table 1.

- It uses a roughly selected eye area for recognition, without executing a specific process for detecting the pupil and iris boundary, the eyelid, and the eyelashes, as this process has a long processing time and its performance is affected by the image quality. To prevent a decrease in recognition performance due to the roughly selected eye area, an ocular area that is somewhat larger than the iris area is used to perform recognition. In addition, it uses a deep ResNet to resolve the problem of misalignment between the enrolled and recognition images that can occur due to recognition based on a roughly detected eye area, as well as the problem of reduced recognition performance caused by this misalignment.

- We analyze the feature maps from various convolutional layers of the deep ResNet in order to examine the features that are important for ocular recognition in each of the layers.

- We make our algorithm for rough pupil detection and ResNet models trained with three open databases (Dongguk CNN Model for NIR Ocular Recognition (DC4NO)) available to other researchers for the fair comparisons as shown in [39].

\section{Proposed Method}

\subsection{Overall Procedure of Proposed Ocular Recognition Method}

Figure 1 shows an overall flowchart of the algorithm proposed in this study. A sub-block based template matching method is used to find a rough pupil area from the input iris image captured by the NIR camera sensor (Step 2 of Figure 1).

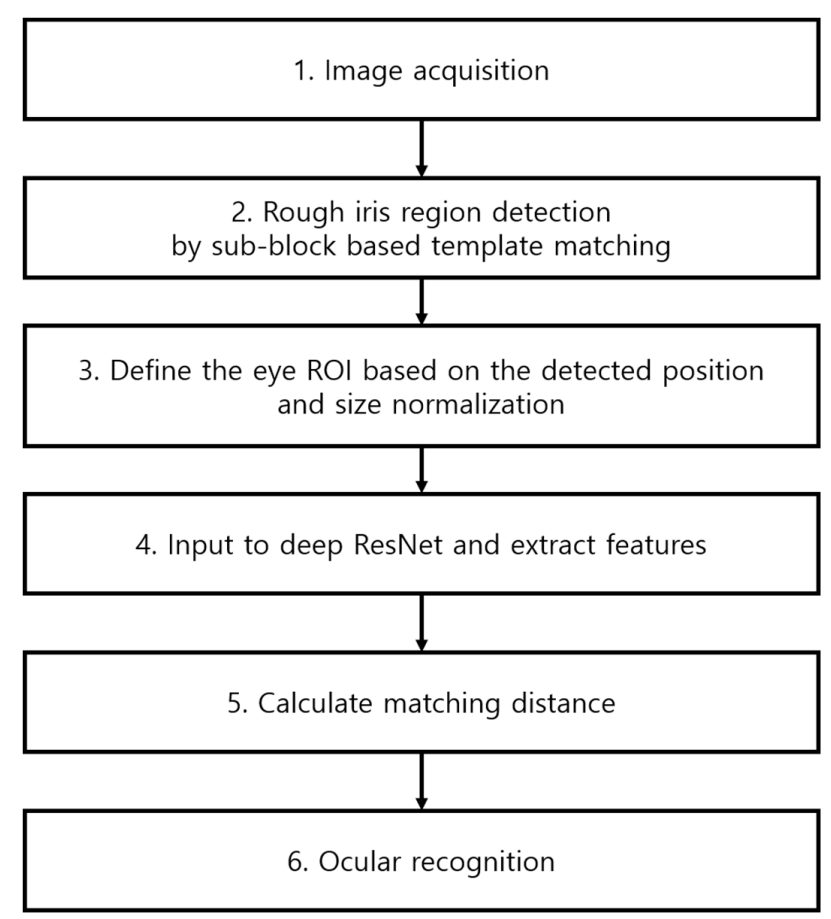

Figure 1. Overview of the proposed system.

A somewhat larger ocular $\mathrm{ROI}$ is specified based on the selected rough pupil location, and a size normalization to $224 \times 224$ pixels is performed on this ROI to use it as input in the deep ResNet that is used in this study (Step 3 of Figure 1). In the next step, the feature vectors of the extracted input ROI 
are extracted from the deep ResNet (Step 4 of Figure 1). The matching distance to the pre-enrolled feature vectors is determined (Step 5 of Figure 1). If this distance is below a defined threshold, the input image is recognized as being in the same class as the pre-enrolled image (acceptance as genuine class). If the distance is greater than the threshold, the input image is rejected and considered as being in a different class from the pre-enrolled image (rejection as imposter class) (Step 6 of Figure 1). Sections 4.2-4.4 provide a more detailed description of this.

\subsection{Rough Pupil Detection and Defining Ocular ROI}

This study performs sub-block-based template matching to find a rough iris area in the input iris images. This algorithm uses a sub-block based template to find a rough eye area. The central sub-block is set as the initial search location, and eight sub-block areas are set in the up, down, left, right, and diagonal directions. After this, the mean values of the pixels with the areas in the up, down, left, right, and diagonal directions are compared. If the center value is not the lowest value, the algorithm moves to the next search area, and the process is repeated to find the location with the lowest value [40]. Moreover, to quickly search an image with this process, an integral image is calculated, and processing is performed based on this [41]. The sum of the area's pixels obtained in this way is calculated for nine sub-blocks, as shown in Figure 2. Then, the position where the mean pixel value of the central sub-block in a certain location and the mean pixel value of its surrounding eight sub-blocks become the minimum vale is determined as the rough pupil location.

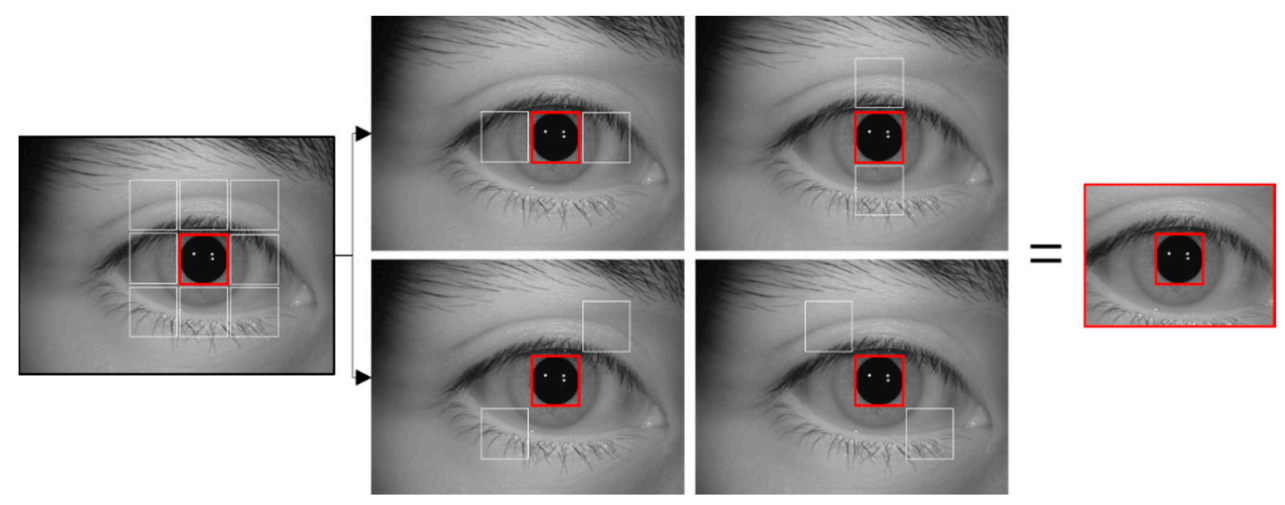

Figure 2. Example of rough iris area detection using sub-block-based template matching.

Although we used the sub-block-based template matching using $3 \times 3$ sub-blocks whose concept is similar to that in [40], there is novel enhancement in our research as follows. In [40], they calculate the difference between the mean of central sub-block and those of surrounding eight sub-blocks at the same time, which increases the processing time. However, in our method, the difference between the mean of central sub-block and those of surrounding two sub-blocks is checked sequentially. In details, the difference between that of central sub-block and those of two horizontal sub-blocks is checked as the first step, and the pupil candidate regions where this difference is small are predicted. Then, with these candidates, the difference between that of central sub-block and those of two diagonal sub-blocks is checked as the second step, and this procedure is repeated for the remained surrounding sub-blocks as shown in Figure 2. Based on this sequential and hierarchical step, the processing speed of our sub-block-based template matching is much enhanced compared to [40].

To consider the cases in which the size of the pupil area varies within the input image due to differences in the z-distance from the camera to the user's eye, the sub-block's size is altered, and the search is adaptively performed. In detail, the initial size of sub-block is $30 \times 30$ pixels with the adaptation to maximum $100 \times 100$ pixels. The stride is constant as 1 pixel in all the cases of sub-block-based template matching. The minimum value of sub-block template matching is calculated by comparing the previously saved minimum value (the difference between the mean of central sub-block and those of surrounding two sub-blocks at previous position) with the value calculated 
at current position. For example, if the value at the current position is smaller than that at previous position, the minimum value is updated by that at the current position whereas it is not updated if not as shown in Equation (1):

$$
D V_{\text {min }}=D V_{c u r}\left(\text { if } D V_{c u r}<D V_{\text {prev }}\right)=D V_{\text {prev }}\left(\text { else if } D V_{c u r} \geq D V_{\text {prev }}\right)
$$

where $D V_{\text {cur }}$ and $D V_{\text {prev }}$ are the difference values between the mean of central sub-block and those of surrounding two sub-blocks at current and previous positions, respectively. $D V_{\min }$ is the minimum difference value. The step of increasing sub-block size is constant as 10 pixel in all the cases of sub-block-based template matching. If the condition is fulfilled for many sizes of sub-blocks, only one sub-block size is determined, which shows the minimum difference value of Equation (1) although it is sub-optimal case. That is because we do not intend to detect accurate ROI for recognition, and the detected ROI is not ideal and has a little positional errors. However, these could be compensated by our deep CNN.

Afterward, an ocular ROI for the CNN input is defined based on the found pupil area, and this ROI is resized via bilinear interpolation. This kind of strategy of rough pupil detection and defining ocular ROI for deep CNN belongs to our proposed method.

Of the three open databases used in this study, the CASIA-Iris-Distance database includes a large face area that includes both eyes in the input images as shown in Figure 3. As such, false detection cases, in which areas outside of the eye are improperly detected, can occur during eye detection that uses the described sub-block-based template matching alone. To resolve this problem, the present study uses the Adaboost eye detector in a search region that includes both eyes in the input image, as shown in Figure 3. The pupil and ocular ROIs are found within this region through sub-block-based template matching.

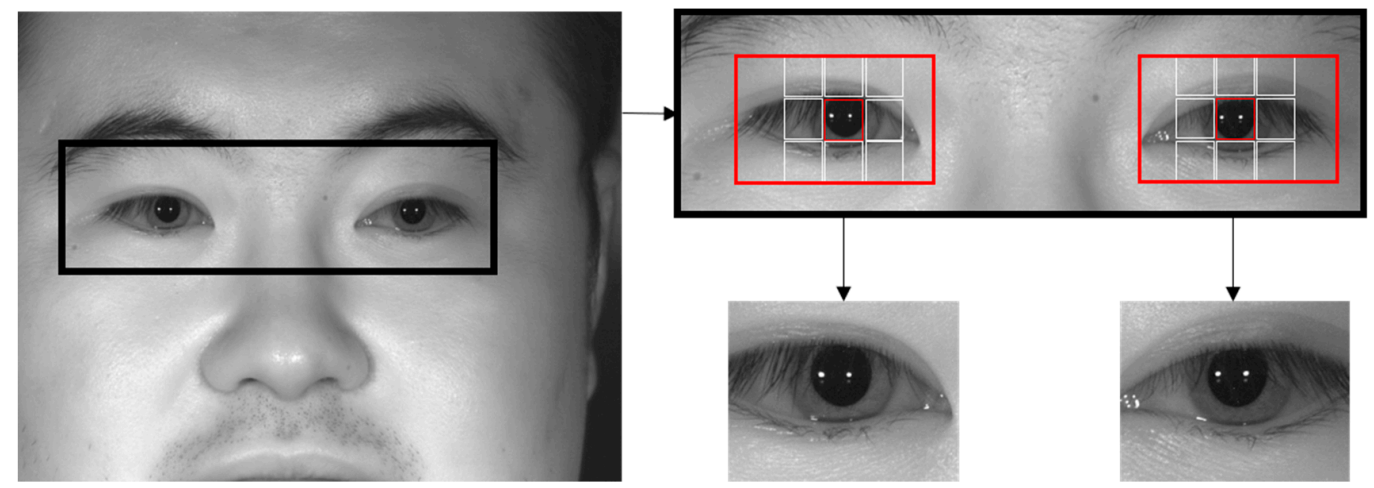

Figure 3. Example of rough detection of ocular ROI in CASIA-Iris-Distance database.

\subsection{Deep ResNet-Based Ocular Recognition.}

The existing CNN architectures that have been studied have demonstrated very good performance in the field of image processing and recognition. Various CNN models have been introduced, including AlexNet by Krizhevsky et al. [42], which comprises five convolutional layers (CL) and three fully connected layers (FCL), and Visual Geometry Group (VGG)-Net by Simonyan et al. [43], which comprises 16 or 19 CLs and three FCLs. Through these studies, it was discovered that performance improves as the layer depth increases. The architecture used in this study, deep ResNet, typically has 50,101, or 152 layers [44]. If the depth of the layers increases without any supplementation, the training accuracy decreases when a learning is performed, and the model cannot converge on the global minimum. This is because features are lost as the model continues to go through processing and the existing image features are reduced through continued calculations. A previous study [44] resolves this problem using the concepts of short-cut (skip-connection) based residual blocks and identity mapping, as shown in Figure 4. 


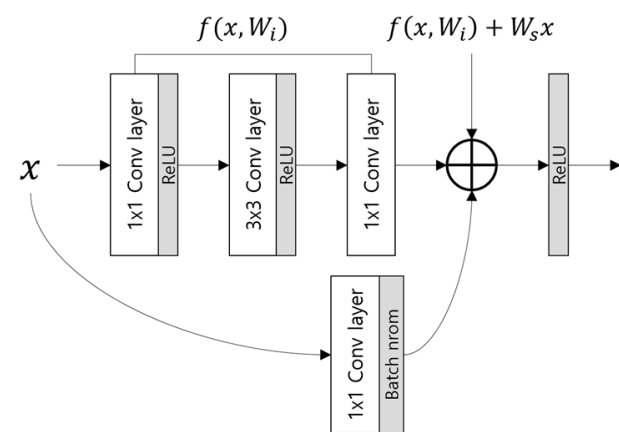

(a)

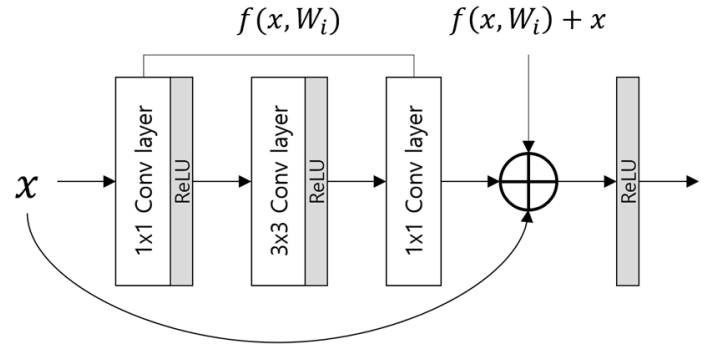

(b)

Figure 4. Examples of processes which involve short-cuts and identity mapping. (a) A process in which a $1 \times 1$ convolution (Conv) is used and the image size is adjusted to adjust the feature map channel when first starting to change the feature map size when processing each residual block. (b) A process that uses identity mapping when repeating the other residual blocks. In (a) and (b), rectified linear unit (ReLU) and batch normalization layer (batch norm) are included.

As seen in Figure 4, elemental-wise addition is performed via the short-cut to identity-map input $x$ to the output where input $x$ is processed through the number of convolution layers $f(x)$. By doing so, the identities of the features that are continually calculated can be preserved even as many layers accumulate. The convolution layers calculated at the same time as this single skip-connection are bound together and called a single residual block. ResNet is based on these residual blocks, and each convolution layer is created from several residual blocks [44]. Table 2 shows a detailed description of the model used in this study for ResNet. In Table 2, Input shows the input layer; Conv shows the convolutional layer; and Max-pool shows the max pooling layer.

As shown in Table 2, each convolution layer comprises an accumulation of residual blocks. Each residual block comprises convolution layers with three small filter sizes of $1 \times 1,3 \times 3$, and $1 \times 1$, and this is called a bottleneck design. It performs fewer calculations than processing two $3 \times 3$ convolutions, and the performance is similar or better [44]. To reduce the output feature map size to half when each convolution layer starts as the residual blocks accumulate, the stride of the block's first $1 \times 1$ convolution layer is set to 2 to reduce the feature map size, and the strides of the other blocks are set to 1 to maintain the feature map size as it is. Likewise, in the case of the shortcut, a $1 \times 1$ conv $+\mathrm{BN}$ is performed in the first shortcut to match the number of channels in order to reduce the feature size of each convolution layer. In the other shortcuts, the $1 \times 1$ conv is not performed and identity mapping which preserves the features is performed through an elemental-wise summation of the output of the previous block as it is. Figure 5 shows the residual blocks in more detail. Conv2_1 refers to the first block of Conv2 in Table 2. The three items in this block, Conv 2A, 2B, and 2C, refer to the $1 \times 1,3 \times 3$, and $1 \times 1$ filter sizes and convolutions, respectively. The scale in Figure 5 shows the magnification operation of the feature map value.

To train with the dataset used in this study, fine-tuning was performed based on the pretrained ResNet model. This is because hundreds of datasets are needed to train the weights of the many layers shown in Table 2, and the test dataset used in this study is inadequate for this. In this study, the pretrained ResNet model of He et al. [44] was used. This model was pre-trained using the ImageNet database, and this database comprises millions of images [45]. The model was used in the ImageNet large scale visual recognition competition (ILSVRC). Because of this, the size of the images was resized to the ImageNet data input size during the input data resizing process in this study. For fine-tuning at this time, the layers that were to be re-trained were selected. In this study, only the fully connected layer part of Conv5 in Table 2 was fine-tuned. 
Table 2. Detailed description of each layer in our deep ResNet $\left(1\left(2^{*}\right)\right.$ means that a stride of 2 is used only when processing each first residual block where the feature map size is reduced to half; otherwise 1 is used) $\left(2^{* *}\right.$ means that it is applied only to a $1 \times 1$ convolution, and it is not applied to identity mapping) (during each iteration, $1 \times 1$ convolution + batch normalization $(\mathrm{BN})$ is performed only during the first iteration, and identity mapping is performed during the other iterations) (Conv3-Conv5 include bottleneck structures).

\begin{tabular}{|c|c|c|c|c|c|c|}
\hline Layer Name & $\begin{array}{c}\text { Size of Feature Map } \\
\text { (Height } \times \text { Width } \times \\
\text { Channel) }\end{array}$ & $\begin{array}{l}\text { Number } \\
\text { of Filters }\end{array}$ & $\begin{array}{l}\text { Size of } \\
\text { Filter }\end{array}$ & $\begin{array}{l}\text { Number of } \\
\text { Padding }\end{array}$ & $\begin{array}{l}\text { Number of } \\
\text { Strides }\end{array}$ & $\begin{array}{c}\text { Number Iteration of } \\
\text { Residual Block }\end{array}$ \\
\hline Input & $224 \times 224 \times 3$ & & & & & \\
\hline Conv1 & $112 \times 112 \times 64$ & 64 & $7 \times 7 \times 3$ & 3 & 2 & 1 \\
\hline Max-pool & $56 \times 56 \times 64$ & 1 & $3 \times 3 \times 1$ & 0 & 2 & 1 \\
\hline $\begin{array}{l}\text { Conv2 } \\
\text { (short-cut) }\end{array}$ & $\begin{array}{l}56 \times 56 \times 256 \\
56 \times 56 \times 256 \\
\end{array}$ & $\begin{array}{c}64 \\
64 \\
256 \\
256 \\
\end{array}$ & $\begin{array}{l}1 \times 1 \times 64 \\
3 \times 3 \times 64 \\
1 \times 1 \times 64 \\
1 \times 1 \times 64 \\
\end{array}$ & $\begin{array}{l}0 \\
1 \\
0 \\
0\end{array}$ & $\begin{array}{l}1 \\
1\end{array}$ & 3 \\
\hline $\begin{array}{l}\text { Conv3 } \\
\text { (short-cut) }\end{array}$ & $\begin{array}{l}28 \times 28 \times 512 \\
28 \times 28 \times 512\end{array}$ & $\begin{array}{l}128 \\
128 \\
512 \\
512\end{array}$ & $\begin{array}{l}1 \times 1 \times 256 \\
3 \times 3 \times 128 \\
1 \times 1 \times 128 \\
1 \times 1 \times 256\end{array}$ & $\begin{array}{l}0 \\
1 \\
0 \\
0\end{array}$ & $\begin{array}{c}1\left(2^{*}\right) \\
1 \\
2^{* *}\end{array}$ & $\begin{array}{l}\text { - } 4 \text { in case of ResNet-50 } \\
\text { and ResNet-101 } \\
\text { - } 8 \text { in case of ResNet-152 }\end{array}$ \\
\hline $\begin{array}{l}\text { Conv5 } \\
\text { (short-cut) }\end{array}$ & $\begin{array}{l}7 \times 7 \times 2048 \\
7 \times 7 \times 2048\end{array}$ & $\begin{array}{c}512 \\
512 \\
2048 \\
2048\end{array}$ & $\begin{array}{l}1 \times 1 \times 1024 \\
3 \times 3 \times 512 \\
1 \times 1 \times 512 \\
1 \times 1 \times 1024\end{array}$ & $\begin{array}{l}0 \\
1 \\
0 \\
0\end{array}$ & $\begin{array}{c}1\left(2^{*}\right) \\
1 \\
2^{* *}\end{array}$ & 3 \\
\hline $\begin{array}{l}\text { Average pooling } \\
\text { layer }\end{array}$ & $1 \times 1 \times 2048$ & 1 & $7 \times 7 \times 1$ & 0 & 1 & 1 \\
\hline $\begin{array}{l}\text { Fully connected } \\
\text { layer (Softmax) }\end{array}$ & $1 \times$ number of classes & & & & & 1 \\
\hline
\end{tabular}

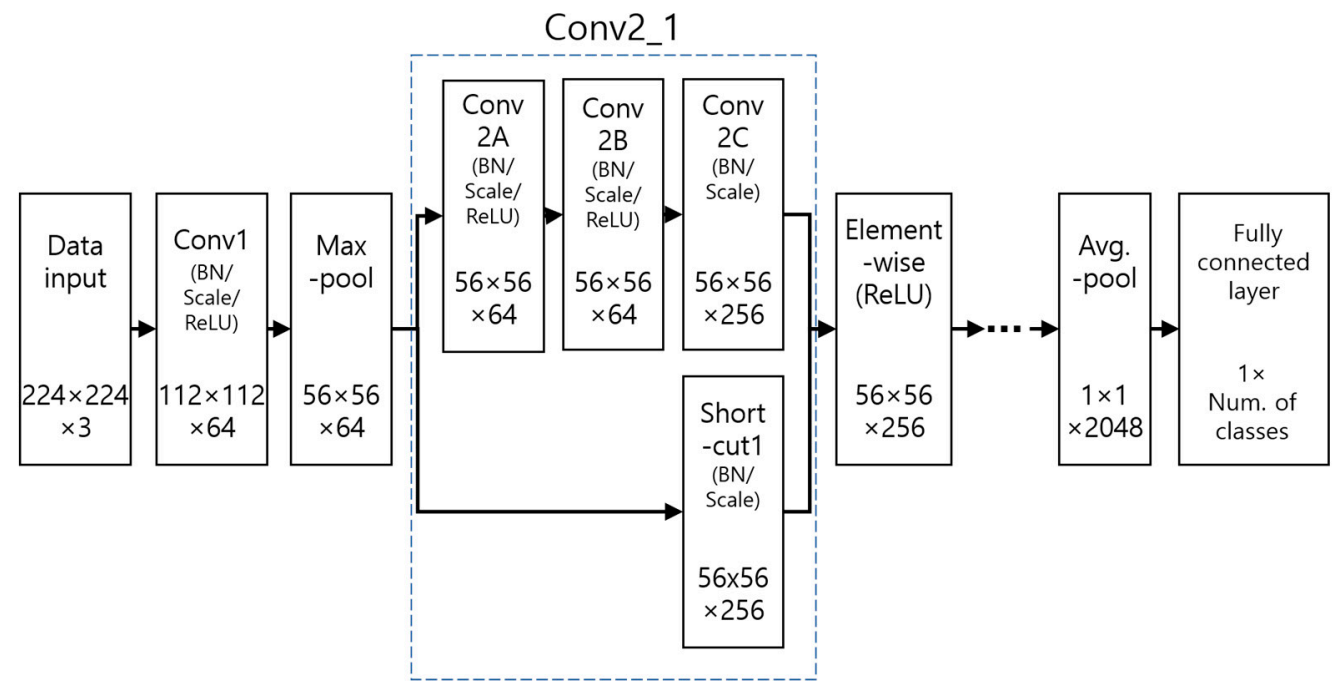

Figure 5. Detail description of residual block.

To train a CNN, several hyper-parameters and optimizers must be selected. Most training processes can be divided into forward processes and backward processes. A forward process initializes a given weight and uses this to perform calculations according to the model's stages in order. Afterward, the ground-truth value that was originally attempted to obtain in the backward process and the results calculated by the forward process are compared to calculate the loss. This error is used and the weights are adjusted going backward to perform training. In the forward process, the activation function is considered important. Previously, a sigmoid function was normally used, but this required a lot of time 
and computation to calculate, so now a ReLU [46] function is usually used as the activation function. This ReLU function is often used because it is easy to calculate and does not produce negative values, and it shows somewhat better performance in making the training converge. Moreover, it does not require considerable computation to calculate the slope value for training [42-44]. The output obtained after the forward process is performed is compared with the ground-truth value, and a backpropagation process is performed to modify the weights using the stochastic gradient descent (SGD) method to optimize the weights of the training model. The first item which must be calculated at this time is the loss between the current results and the ground truth. How accurately this can be calculated determines whether the process will converge so that the training is completed properly. For the method used here, calculations were performed through basic multinomial logistic loss, and the output predicted through softmax [47] was used to perform the calculation. If the calculations are performed in this manner, it is possible to maintain more numerical stability when calculating the slope. Each of the results calculated by softmax function is used as an input to calculate the multinomial logistic loss [48].

\subsection{Extracting Feature Vector and Calculating Matching Distance}

In normal biometric studies, recognition performance is measured under two settings: closed world and open world. In the former, the classes of data are the same during training and testing. In the latter, the classes of data are different during training and testing. Under normal scenarios in biometrics, the classes of data may or may not be the same during training and testing. Therefore, an open world setting is more suitable for real-world applications. This study evaluated recognition performance in an open world setting. During this type of classification for biometrics, the output of the CNN's fully connected layer is used, or the feature vectors extracted from the layer before the last fully connected layer are used to perform matching based on the matching distance with the feature vectors of the enrolled images. In a closed world setting, the classes of the data are the same during training and testing. Therefore, the output of the CNN's fully connected layer can be used as-is. However, in an open world setting, the classes of data are different during training and testing. Therefore, feature vectors extracted from the layer before the last fully connected layer are used to perform recognition based on the matching distance with the feature vectors of the enrolled images. This study obtained the matching distance using Euclidean distance based on 2048 feature vectors extracted from Table 2's average pooling layer. If the enrolled and input images are in the same class (genuine matching), a small Euclidean distance is calculated for them. Conversely, if they are in different classes (imposter matching), a large Euclidean distance is calculated for them. In this study, genuine matching occurred when this Euclidean distance was smaller than the threshold, and imposter matching occurred when it was larger than the threshold. Based on the training data, the distance at which the false acceptance error (FAR) and the false rejection error (FRR) were the same was set as the optimal threshold. FAR is the error of incorrectly accepting imposter data as genuine data, whereas FRR is that of incorrectly rejecting genuine data as imposter data. In general, FAR and FRR share a trade-off relationship, and the error in case FAR is similar to FRR is called as the equal error rate (EER).

\section{Experimental Results with Analysis}

\subsection{Datasets and Data Augmentation}

To evaluate the performance of the ocular recognition method proposed in this study, three types of open databases captured in an NIR camera environment were used to perform tests: CASIA-Iris-Distance, CASIA-Iris-Lamp, and CASIA-Iris-Thousand databases [49]. The distances between camera and user's eye in case of collecting CASIA-Iris-Lamp and CASIA-Iris-Thousand databases are also in rear range, which are similar to that of CASIA-Iris-Interval. Therefore, the influence by NIR sources on the average illumination level of the center block was already tested in our experiments. Each of the databases was divided into two subsets, and two-fold cross validation 
was performed. For example, the CASIA-Iris-Distance database's 282 classes, which include both eyes of the 141 people, were divided into sub-database 1 (DB1) and sub-database 2 (DB2) with 71 (142 classes) and 70 people (140 classes), respectively.

Data augmentation was performed, and then training was conducted. The data augmentation is to increase the number of training data. The augmented data were used only for training, and original data were used for testing. In this way, training and testing were performed separately for two-fold cross validation. By doing so, the study aimed to prevent the problem of insufficient training data and overfitting in which the CNN trains excessively for the training data and the performance for the testing data is reduced. The mean accuracy obtained from two rounds of testing based on two-fold cross validation was used as the ultimate accuracy of the method proposed in this study. Table 3 below contains detailed descriptions of the experimental databases used in this study.

Table 3. Detail descriptions of experimental databases.

\begin{tabular}{ccccccc}
\hline \multirow{2}{*}{ Category } & \multirow{2}{*}{ Number of Classes } & \multicolumn{4}{c}{ Number of Images } \\
\cline { 2 - 7 } & \multicolumn{1}{c}{ DB1 } & DB2 & DB1 & DB2 & DB1 & DB2 \\
\cline { 2 - 6 } & 142 & 140 & 2080 & 2056 & 351,520 & 347,464 \\
\hline CASIA-Iris-Distance & 408 & 408 & 8054 & 8036 & $1,361,126$ & $1,358,084$ \\
\hline CASIA-Iris-Lamp & 1000 & 1000 & 9946 & 9946 & $1,680,874$ & $1,680,874$ \\
\hline CASIA-Iris-Thousand & & & & & & After Augmentation \\
\hline
\end{tabular}

For the training data, data augmentation was performed on the ROI areas found through the sub-block based template matching described in Section 4.2. Six pixels of translation and cropping in the up, down, left, and right directions were performed as shown in Figure 6 to augment the data by a factor of 169 . Such translation and cropping-based data augmentation has been widely used in previous studies [42]. Through this, the misalignment between the enrolled and recognition images can be covered through CNN training, and the problem of inadequate training due to a small dataset can be resolved.
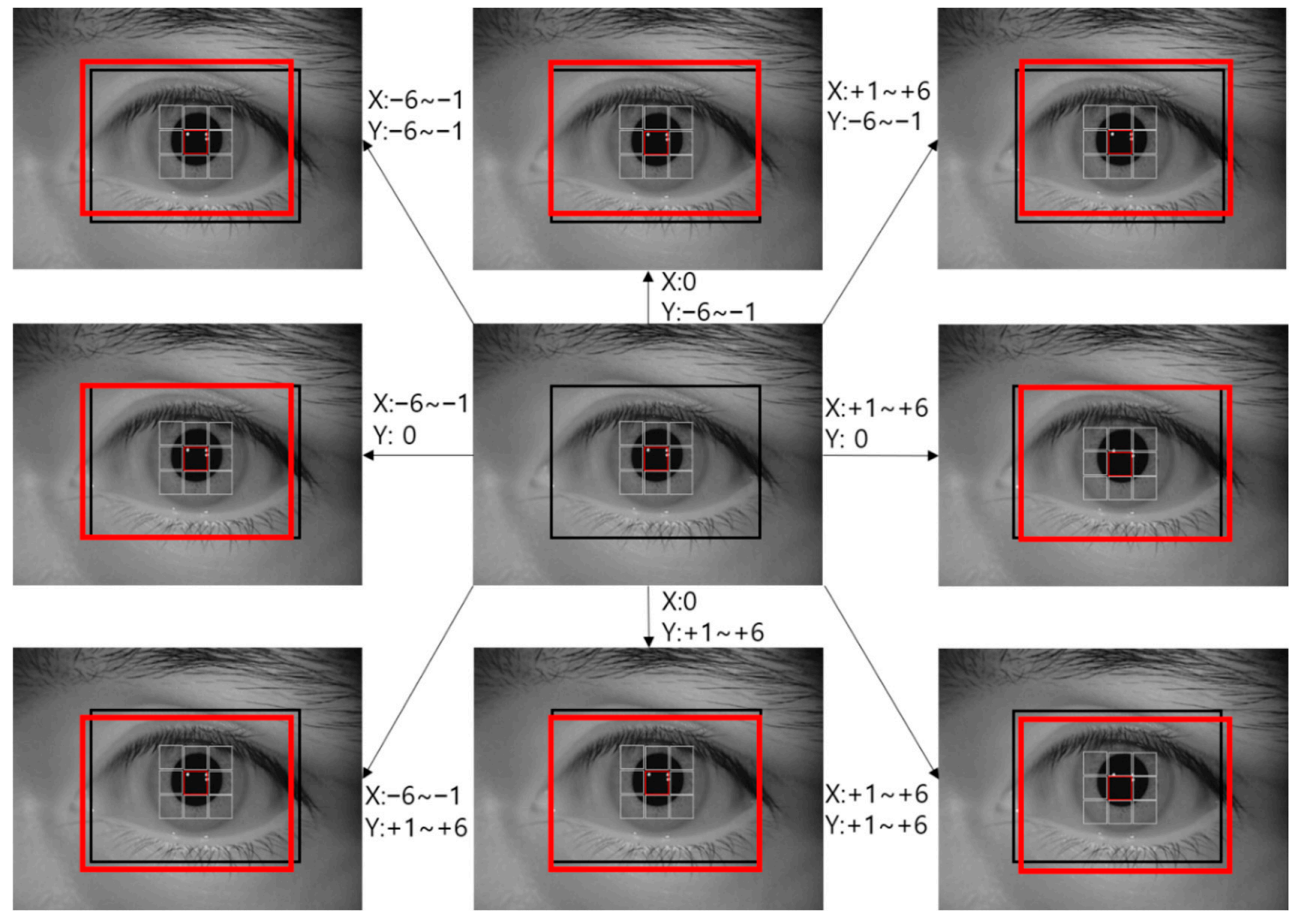

Figure 6. Example of data augmentation process used in this study. 


\subsection{Training of CNN Model}

In the tests, the ResNet-50, 101, and 152 models were used to perform fine-tuning via the described augmented training data. During training, a stochastic gradient descent (SGD) optimizer [50] was used. Here, optimization was performed using a step policy as the learning rate policy, in which the gamma value was multiplied every fixed iteration. As one of SGD's features, training was performed in the unit of mini-batch sizes. The number of iterations was calculated as the "number of training data/mini-batch size," and the number of iterations here was defined as 1 epoch. In this study, training was performed for 3-10 epochs for each model. The learning rate was 0.0001. A small learning rate was used because fine-tuning was being performed using existing learned weights; the momentum value was 0.9 , weight decay was 0.0001 , and the gamma value was 0.1 . Because the number of images in each dataset varied, the number of steps varied to match this and find optimal performance. To calculate the training loss, the multinomial logistic loss was calculated using the softmax function. Figure 7 below shows graphs of the training loss and training accuracy obtained during the training of ResNet-50, 101, and 152. As shown in Figure 7, as the training iterations increase, the training loss converges near 0 and the training accuracy converges near 100\%. This shows that the training of the CNN model used in this study was performed successfully.

Training and testing for the method proposed in this study were performed on a desktop computer equipped with an Intel i7-975 3.33 GHz, 16 GB of RAM, and an NVIDIA GTX1070 graphic processing unit (GPU) [51]. The compute unified device architecture (CUDA) (version 8.0) and CUDA deep neural network library (cuDNN) (version 5.0) environments were used. Algorithms were implemented in OpenCV (version 3.3.0), Visual Studio 2015, and Windows Caffe (version 1.0.0) [52].
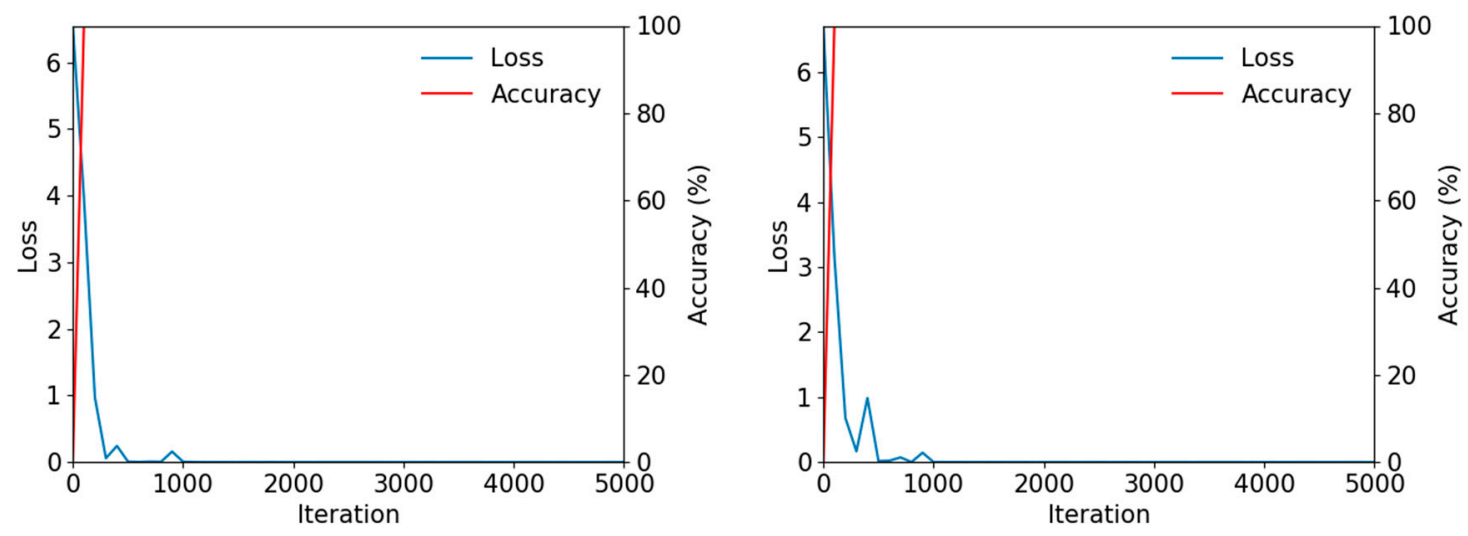

(a)
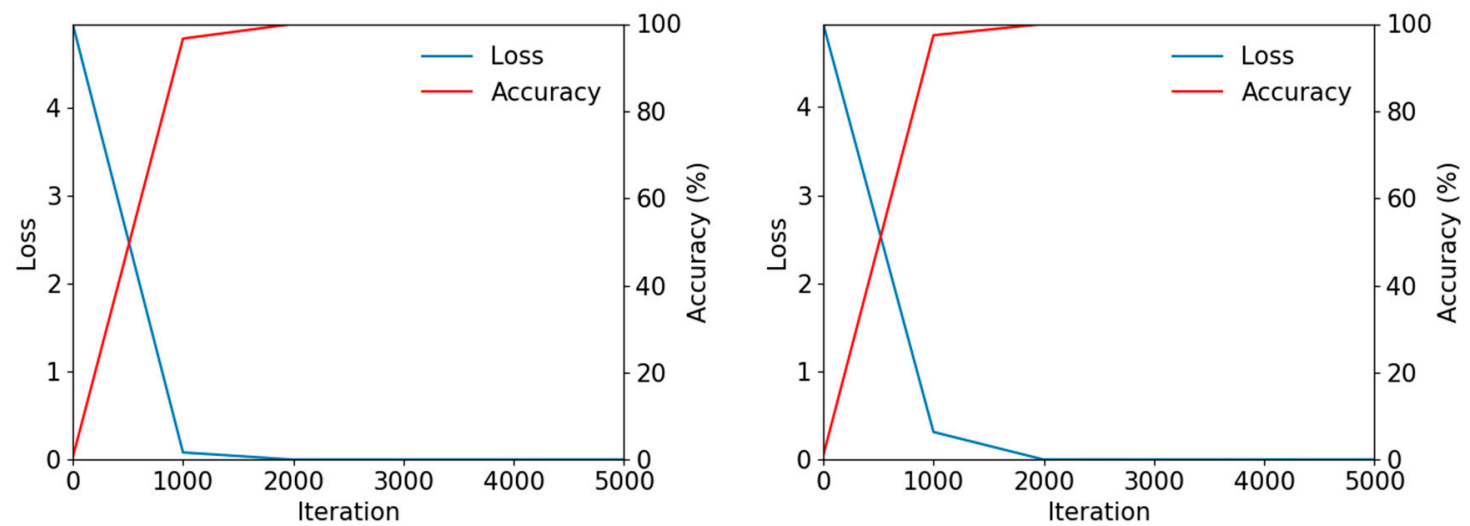

(b)

Figure 7. Cont. 

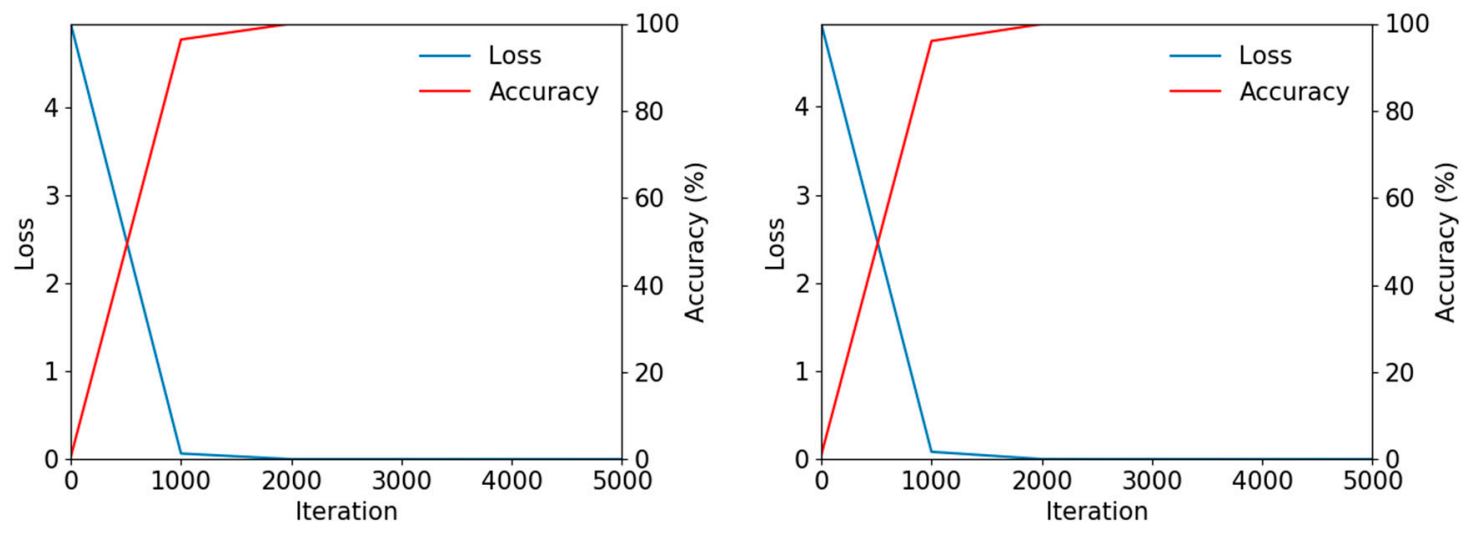

(c)

Figure 7. Examples of training loss and accuracy graphs with CASIA-Iris-Distance database. Left and right figures are the graphs of DB1 and DB2 training, respectively. Graphs of training by (a) ResNet-50,

(b) ResNet-101 and (c) ResNet-152 models, respectively.

\subsection{Testing of Proposed CNN-Based Ocular Recognition}

For the first test, the recognition accuracy of a variety of ResNet models was measured for each of the test databases. As described in Section 5.1, the first-fold validation (DB1), second-fold validation (DB2), and mean accuracy were found to be in accordance with the two-fold cross validation. The recognition accuracy was measured via the EER described in Section 4.4. There are examples of existing studies in which the accuracy is assessed as the $d^{\prime}$ (d prime) value [53]. The $d^{\prime}$ value is a concept wherein the distance between the genuine and imposter distributions is calculated based on the mean and standard deviations of the two distributions. However, the $\mathrm{d}^{\prime}$ value is generally useful if the genuine and imposter distributions follow a Gaussian distribution, and it is difficult to use the value as an index for measuring accurate recognition performance if these distributions do not follow a Gaussian distribution. In this study, EER was used. As shown in Table 4, ResNet-101 showed the best recognition performance for the CASIA-Iris-Distance database, ResNet-50 for the CASIA-Iris-Lamp database, and ResNet-152 for the CASIA-Iris-Thousand database.

Table 4. Comparative EERs with each dataset by various ResNet architectures (unit: \%).

\begin{tabular}{cccccccccc}
\hline \multirow{2}{*}{ ResNet Model } & \multicolumn{3}{c}{ CASIA-Iris-Distance } & \multicolumn{3}{c}{ CASIA-Iris-Lamp } & \multicolumn{2}{c}{ CASIA-Iris-Thousand } \\
\cline { 2 - 10 } & DB1 & DB2 & Average & DB1 & DB2 & Average & DB1 & DB2 & Average \\
\hline 50-layer & 2.576 & 1.971 & 2.2735 & 1.505 & 1.685 & 1.595 & 2.091 & 2.524 & 2.3075 \\
\hline 101-layer & 2.138 & 2.187 & 2.1625 & 1.702 & 1.738 & 1.72 & 1.431 & 2.570 & 2.0005 \\
\hline 152-layer & 2.103 & 2.264 & 2.1835 & 5.203 & 4.372 & 4.7875 & 1.588 & 1.074 & 1.331 \\
\hline
\end{tabular}

Figure 8 shows the recognition accuracy measured in Table 4 in more detail as a receiver operating characteristic (ROC) curve. The genuine acceptance rate is calculated as $1-F R R$. Each graph is the mean graph of the two graphs found in the two-fold validations. From the results in Figure 8, it is clear that ResNet-101 showed the best recognition performance for the CASIA-Iris-Distance database, ResNet-50 for the CASIA-Iris-Lamp database, and ResNet-152 for the CASIA-Iris-Thousand database.

For the next test, the recognition performance was evaluated according to the size of the eye area ROIs obtained through the method described in Section 4.2. The test was performed on the CASI-Iris-Distance database because the size of the eye area that includes the iris in the CASIA-Iris-Distance database is smaller than that in the other two databases. Therefore, it is expected that the degree to which the area around the eye (periocular area) is included will change considerably, according to the changes in the size of the ROI; this will have a relatively large effect on the recognition 
performance. Based on the results in Table 4, ResNet-101 was used, which shows the highest recognition performance for the CASIA-Iris-Distance database. The average EER of the two-fold cross validation-based testing was found. As shown in Table 5, the recognition EER was the lowest when an ROI of $380 \times 280$ (width $\times$ height) pixels was used. The EER does not change significantly according to the change in the size of ROI in contrast to the recognition EER. From this, it can be known that the method used in this study was not greatly affected by changes in the size of the ROI. When the ROI was $380 \times 400$ (width $\times$ height) pixels, the EER increased further, and this is attributed to the ROI being larger vertically than horizontally, and it including more of the skin area in the vertical direction rather than on both edges of the eye, which are important for recognition. Based on these results, it was found that the EER can be reduced by making the ROI larger horizontally than vertically during recognition. The ROI of $300 \times 260$ shows the high EER, which means that too small ROI cannot include the important features of ocular region and consequent recognition error increases.

Based on [54], we compared the recognition accuracies with pre-trained ResNet without fine-tuning and fine-tuned ResNet on CASIA-Iris-Distance database. The pre-trained ResNet was pre-trained using the ImageNet database, and this database comprises millions of images as explained in Section 4.3 [45]. As shown in Table 6, our fine-tuned ResNet shows the higher accuracy than pre-trained ResNet without fine-tuning.

Table 5. Comparisons in recognition accuracies according to ROI crop size (unit: \%).

\begin{tabular}{cccccc}
\hline & $\mathbf{5 0 0} \times \mathbf{4 0 0}$ Pixels & $\mathbf{5 0 0} \times \mathbf{2 8 0}$ Pixels & $\mathbf{3 8 0} \times \mathbf{4 0 0}$ Pixels & $\mathbf{3 8 0} \times \mathbf{2 8 0}$ Pixels & $\mathbf{3 0 0} \times \mathbf{2 6 0}$ Pixels \\
\hline EER & 2.184 & 2.282 & 3.334 & 2.163 & 2.955 \\
\hline
\end{tabular}

Table 6. Comparative EERs with pre-trained ResNet without fine-tuning and fine-tuned ResNet (unit: \%).

\begin{tabular}{cccc}
\hline Method & DB1 & DB2 & Average \\
\hline Pre-trained ResNet without fine-tuning & 11.504 & 9.728 & 10.616 \\
\hline Fine-tuned ResNet (proposed method) & 2.138 & 2.187 & 2.1625 \\
\hline
\end{tabular}

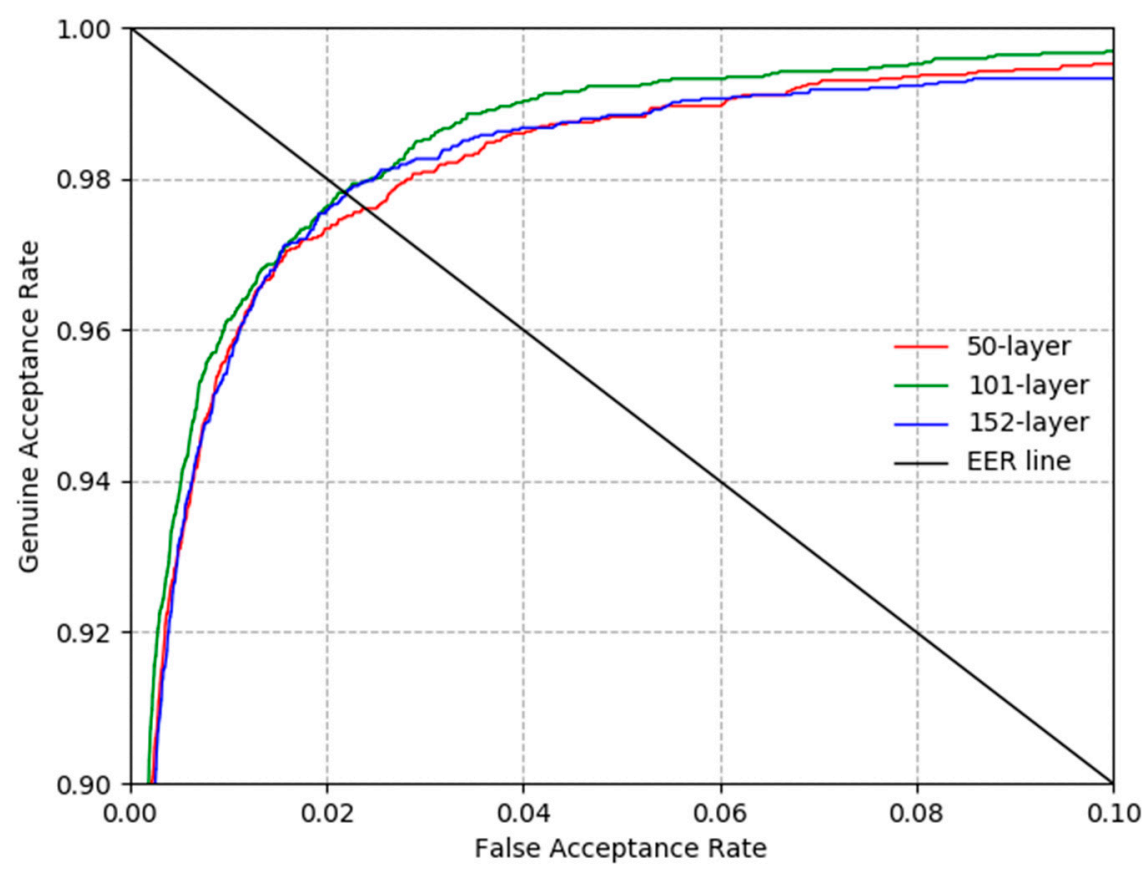

(a)

Figure 8. Cont. 


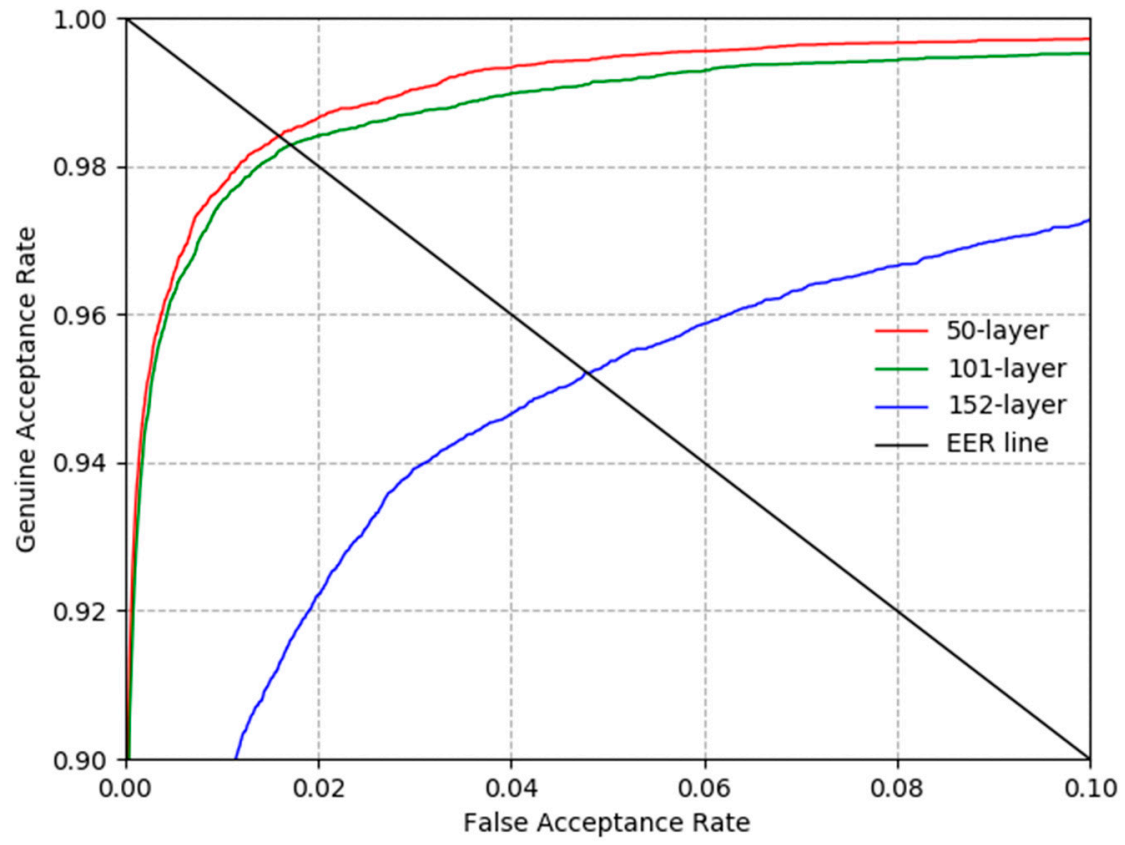

(b)

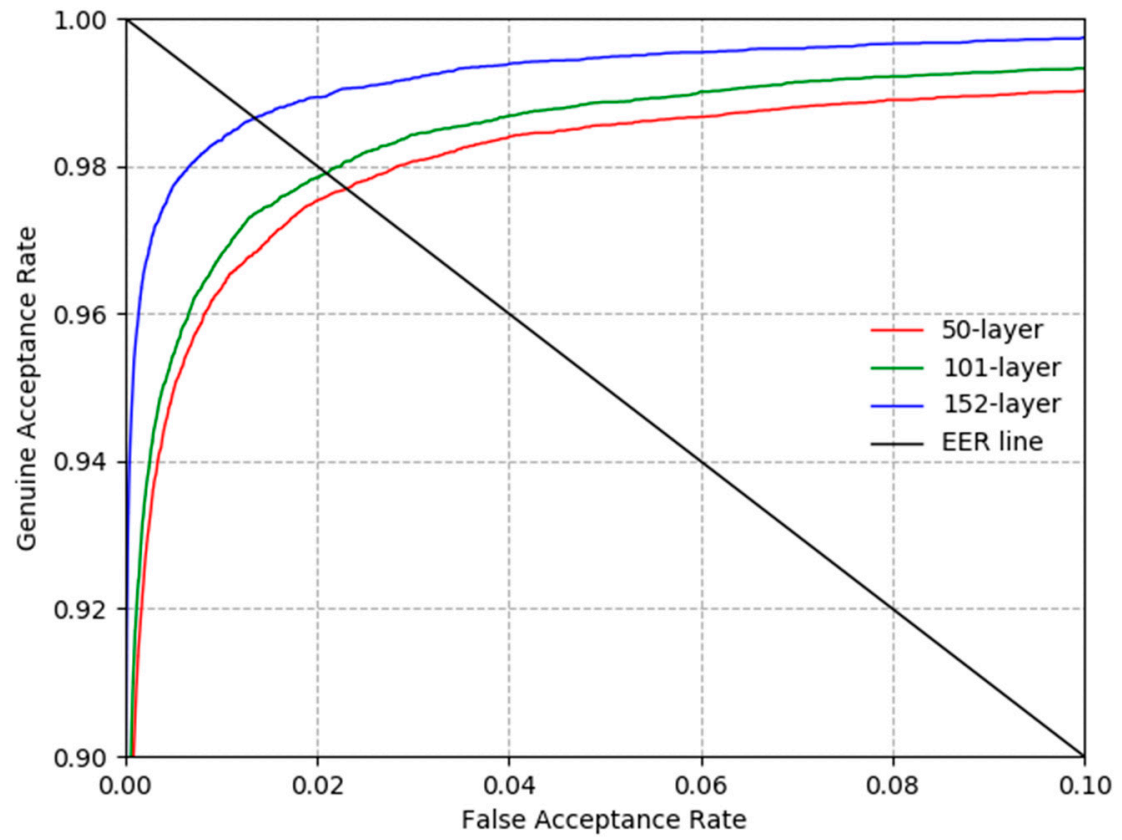

(c)

Figure 8. ROC curves of recognition by ResNet-50, 101, and 152 with (a) CASIA-Iris-Distance, (b) CASIA-Iris-Lamp, and (c) CASIA-Iris-Thousand.

Figure 9 below shows examples of correct recognition cases achieved through the method proposed in this study. As shown in Figure 9, correct recognition occurred even in cases where there were some differences between the enrolled image and the recognition image including the level of eye openness, in-plane rotation, off-angle, scaling, and specular reflection on glasses surface. 

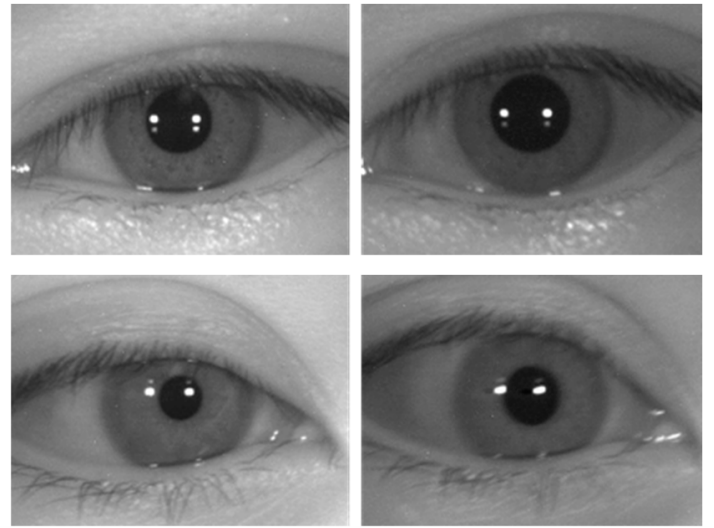

(a)
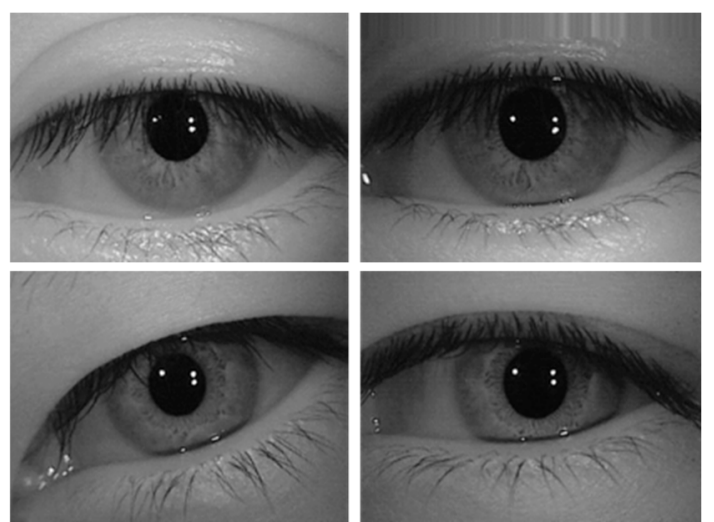

(c)
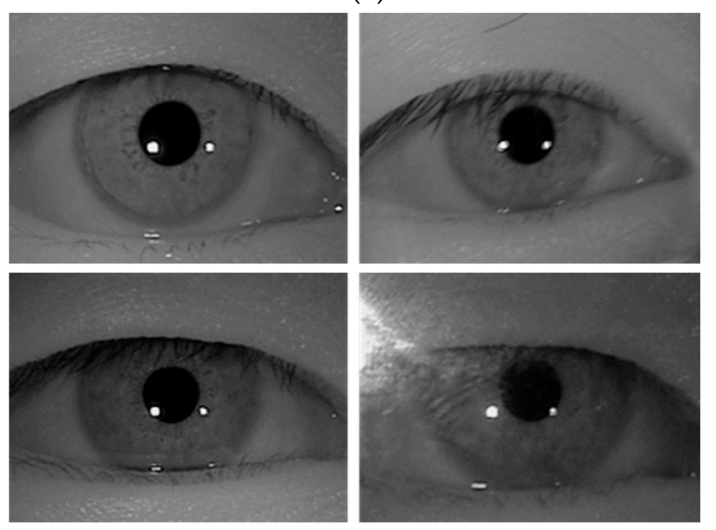

(e)
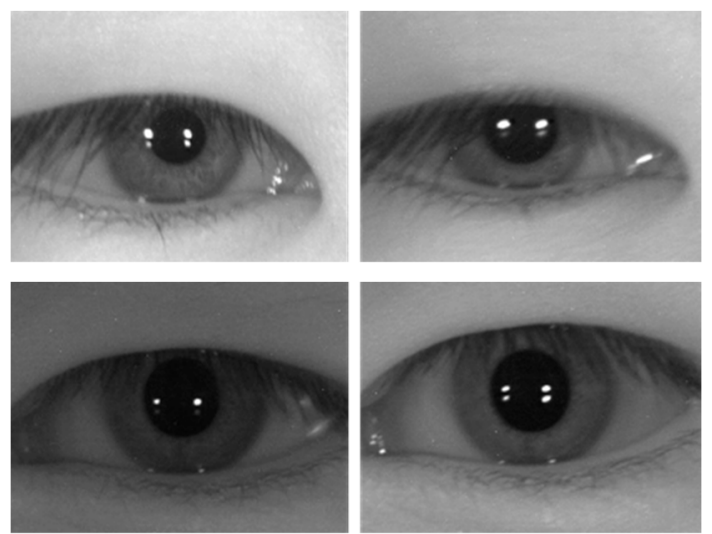

(b)
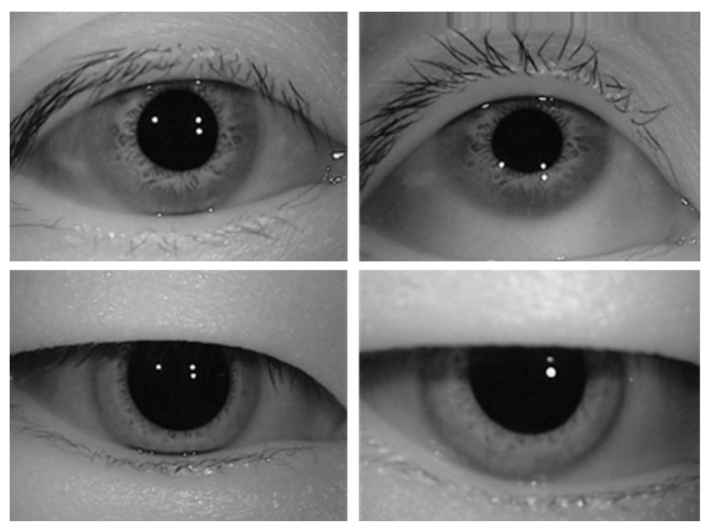

(d)
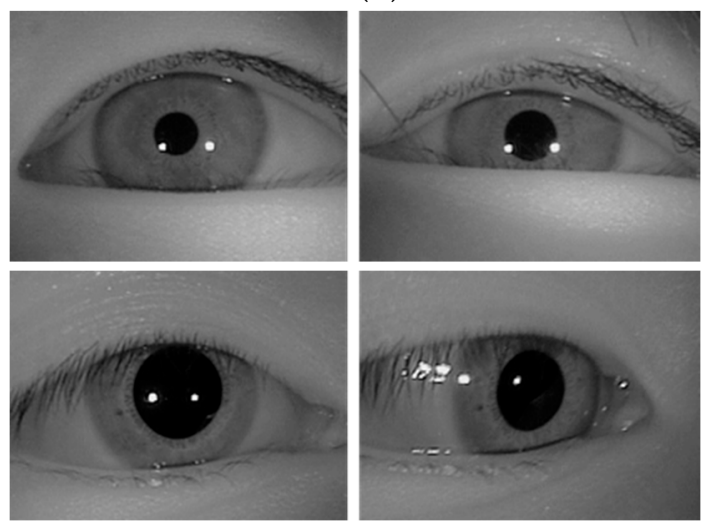

(f)

Figure 9. Examples of correction recognition cases achieved through the method proposed in this study. (a,b) CASIA-Iris-Distance database. (c,d) CASIA-Iris-Lamp database. (e,f) CASIA-Iris-Thousand database. In (a-f), the image on the left is the enrolled image, and the image on the right is the recognition attempt image.

Figure 10 below shows false rejection (FR) and false acceptance (FA) cases that occurred due to the method proposed in this study. As shown in Figure 10a,c,e, FR cases occur when severe in-plane rotation and off-angle happen, or glasses frame is included in the image. As shown in Figure 10b,d,f, FA cases happen when the enrolled and recognition images are similar. 

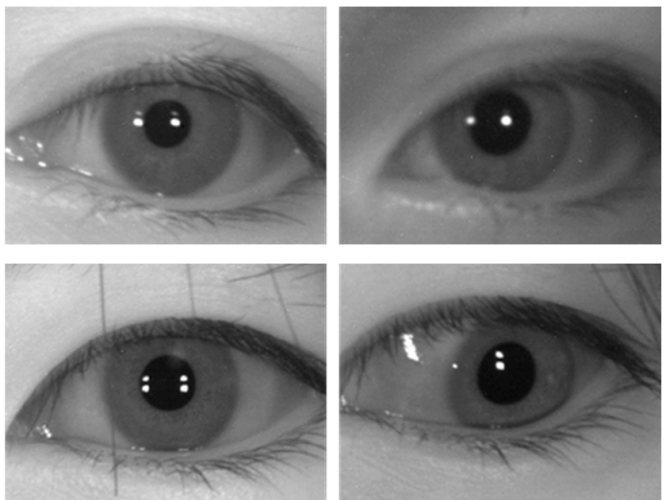

(a)
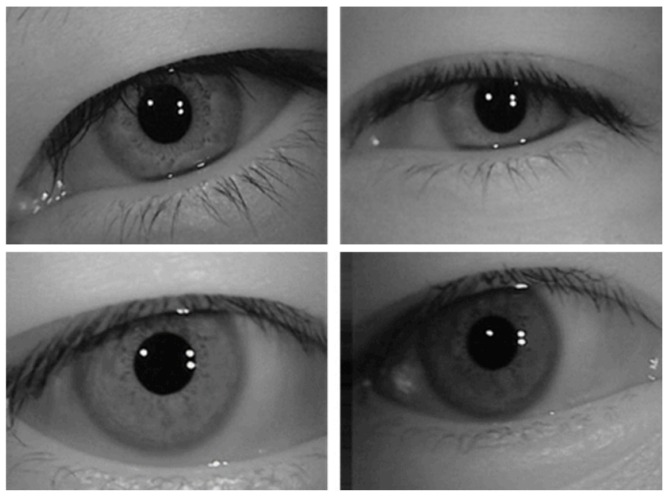

(c)
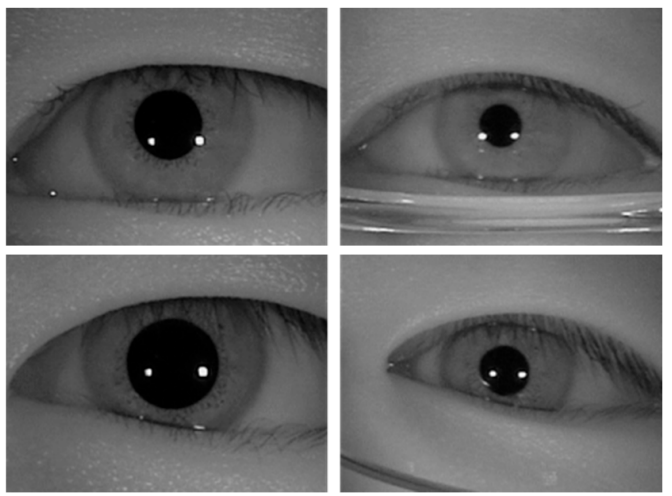

(e)
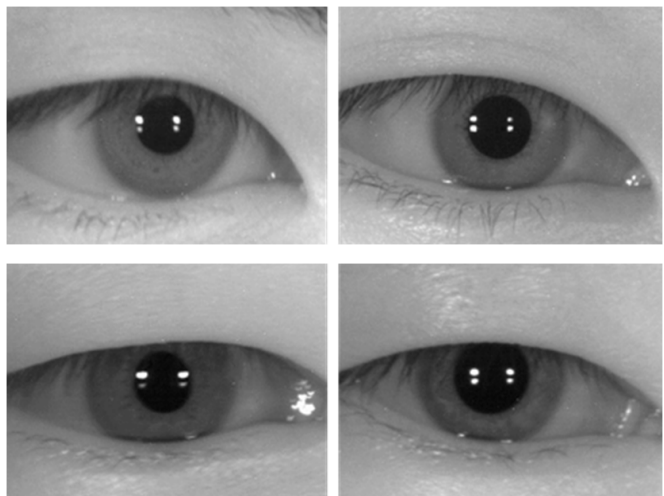

(b)
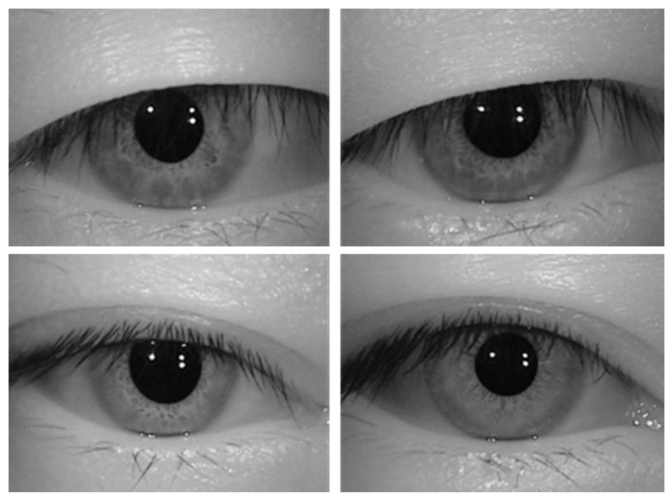

(d)
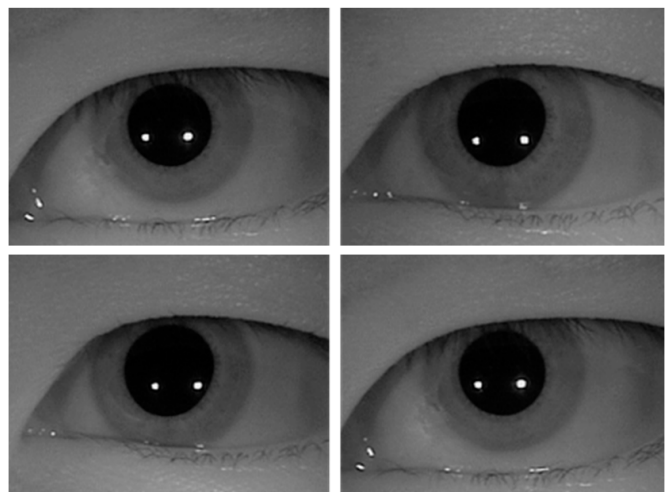

(f)

Figure 10. Examples of recognition error cases due to the method proposed in this study. $(\mathbf{a}, \mathbf{b})$ CASIAIris-Distance database. (c,d) CASIA-Iris-Lamp database. (e,f) CASIA-Iris-Thousand database. (a,c,e) are FR cases. $(\mathbf{b}, \mathbf{d}, \mathbf{f})$ are FA cases. In $(\mathbf{a}-\mathbf{f})$, the image on the left is the enrolled image, and the image on the right is the recognition attempt image.

\subsection{Comparisons with Proposed and Existing Methods}

In the next test, the recognition accuracy of the method proposed in this study and existing methods were compared for the CASIA-Iris-Distance, CASIA-Iris-Lamp, and CASIA-Iris-Thousand databases. In Tables 7-9, the method proposed in this study showed higher recognition accuracy than the existing research methods.

The recognition method that used the deep features extracted from ResNet and the simple ocular area detection method employed by this study showed higher recognition accuracy than the existing handcrafted feature-based method or the traditional machine learning-based method. 
Table 7. Comparative EERs on CASIA-Iris-Distance database (unit: \%).

\begin{tabular}{cc}
\hline Method & EER \\
\hline Cho et al. [20] & 10.0172 \\
\hline Shekar et al. [55] & 8.64 \\
\hline Zhao et al. [30] & 4.9 \\
\hline Shin et al. [8] & 4.3006 \\
\hline Oishi et al. [24] & $3.6 \sim 3.9$ \\
\hline Sharifi et al. [35] & $3.12 \sim 3.465$ \\
\hline Lee et al. [26] & $3.04 \sim 3.08$ \\
\hline Tan et al. [56] & 2.9 \\
\hline Proposed method & 2.1625 \\
\hline
\end{tabular}

Table 8. Comparative EERs on CASIA-Iris-Lamp database (unit: \%).

\begin{tabular}{cc}
\hline Method & EER \\
\hline Uhl et al. [57] & 12.9 \\
\hline Ribeiro et al. [29] & 3.92 \\
\hline Abdullah et al. [58] & 2.37 \\
\hline Proença et al. [59] & 2.6 \\
\hline Nigam et al. [60] & 2.13 \\
\hline Zhang et al. [61] & 2.05 \\
\hline Li et al. [62] & 2.02 \\
\hline Proposed method & 1.595 \\
\hline
\end{tabular}

Table 9. Comparative EERs on CASIA-Iris-Thousand database (unit: \%).

\begin{tabular}{cc}
\hline Method & EER \\
\hline Drozdowski et al. [31] & 8.27 \\
\hline Proença et al. [59] & 3 \\
\hline Li et al. [62] & 2.59 \\
\hline Proposed method & 1.331 \\
\hline
\end{tabular}

As the next experiment, we check whether the recognition performance is related to the part of iris in the ocular image. In previous research [63], they compared the accuracies only by periocular region, iris region, and fusion, respectively. However, the periocular region included both iris and periocular regions in their research, and the accurate effect only by periocular region without iris area was not measured. Therefore, we used the following scheme. With CASIA-Iris-Distance database, the segmented iris regions were painted as black pixels. Then, training and testing with our deep CNN using these images were performed. As shown in Table 10, the recognition error with these images (using periocular region without iris area) is higher than that by proposed method (using whole ocular region). Based on this result, we can find that the recognition performance is related to the part of iris in the ocular image and the iris region is also necessary for ocular recognition of high accuracy. In addition, as shown in Table 10, the accuracy by using iris area without periocular region was measured, and it is lower than that by using whole ocular region. Based on these results, the ocular region including both periocular and iris areas is necessary for high recognition accuracy.

As the next experiment, we performed the experimental comparisons based on the [63]. In this paper, they considered that the periocular region includes both iris and periocular regions. Therefore, 
their method based on the periocular region corresponds to our method using the whole ocular region. As shown in Table 11, our proposed method shows the higher accuracy than those by using iris area without periocular region and the fusion of iris and periocular regions.

Table 10. Comparative EERs on CASIA-Iris-Distance database in case of using whole ocular region or using periocular region without iris area (unit: \%).

\begin{tabular}{cc}
\hline Method & EER \\
\hline Using periocular region without iris area & 5.2506 \\
\hline Using iris area without periocular region & 3.8952 \\
\hline Using whole ocular region (proposed method) & 2.1625 \\
\hline
\end{tabular}

Table 11. Comparative EERs on CASIA-Iris-Distance database for measuring the effect of iris or periocular region based on [63] (unit: \%).

\begin{tabular}{cc}
\hline Method & EER \\
\hline Using iris area without periocular region & 9.5069 \\
\hline Using periocular region (proposed method) & 2.1625 \\
\hline The fusion of iris and periocular regions [63] & 6.2207 \\
\hline
\end{tabular}

In this research, we also performed the experimental comparisons based on the [64]. As shown in Table 12, the method with our augmented database shows the higher accuracy than that with the augmented database based on affine transform [64].

Table 12. Comparative EERs on CASIA-Iris-Distance database in case of training with augmented database based on affine transform [64] with our augmented database (unit: \%).

\begin{tabular}{cc}
\hline Method & EER \\
\hline Training with augmented database based on affine transform [64] & 4.2352 \\
\hline Training with our augmented database & 2.1625 \\
\hline
\end{tabular}

As the next test, in order to include the more variations in augmented data, we performed the additional experiments to compare the accuracy by using our augmented data (explained in Section 5.1) with that by using CASIA-Iris-Thousand database [49] for training our CNN model. The reason why we used CASIA-Iris-Thousand database is that the number of images and classes are the largest among all the CASIA iris databases including CASIA-Iris-Syn database [49]. As shown in Table 13, we can find that the case of training with our augmented database shows the higher accuracy than that by training with CASIA-Iris-Thousand database.

Table 13. Comparative EERs on CASIA-Iris-Distance database in case of training with CASIA-IrisThousand database or training with our augmented database (unit: \%).

\begin{tabular}{cc}
\hline Method & EER \\
\hline Training with CASIA-Iris-Thousand database & 2.5471 \\
\hline Training with our augmented database & 2.1625 \\
\hline
\end{tabular}

For the final test, a processing speed comparison was performed on the sub-block based template matching ocular ROI detection method used in this study, a two-circular edge detector [6], and a CNN-based iris segmentation method [65]. The test environment was described in Section 5.2. As shown in Table 14, it can be seen that the sub-block-based template matching ROI detection method 
used in this study requires less processing time than the existing two-circular edge detector [6] and the CNN-based iris segmentation method [65]. Table 14 also shows the time for performing ocular recognition with the deep ResNet proposed in this study based on the specified ROIs, and the ultimate processing time was found to be $115 \mathrm{~ms}$. From this, it can be known that the recognition system proposed in this study can operate at a speed of about $5.3(=1000 /(73+115))$ frames per second.

Table 14. Comparisons of processing speed by proposed method with other methods (unit: ms).

\begin{tabular}{ccccc}
\hline & $\begin{array}{c}\text { Proposed Method } \\
\text { (Sub-Block Based } \\
\text { Template Matching) }\end{array}$ & $\begin{array}{c}\text { Two-Circular } \\
\text { Edge Detector [6] }\end{array}$ & $\begin{array}{c}\text { CNN-Based Iris } \\
\text { Segmentation [65] }\end{array}$ & $\begin{array}{c}\text { Ocular Recognition } \\
\text { (Feature Extraction + } \\
\text { Distance Matching) }\end{array}$ \\
\hline $\begin{array}{c}\text { Processing time } \\
\text { per an image }\end{array}$ & 73 & 986 & 210 & 115 \\
\hline
\end{tabular}

\subsection{Analysis of Feature Maps Extracted from CNN Convolutional Layers and Discussion}

In general, a $\mathrm{k} \times \mathrm{k} \times \mathrm{d}$ size filter is used on a $\mathrm{w} \times \mathrm{h} \times \mathrm{d}$ size input, as shown in Figure 11a, via a calculation in the convolution layer to find an output feature map of the size $\mathrm{w}^{\prime} \times \mathrm{h}^{\prime} \times \mathrm{Num}$. of output. Here, the number of filters used is the number of output (Num. of output) such that the depth of the ultimate output feature map is the number of output. Figure $11 \mathrm{~b}$ shows an example of convolution calculations and finding the output feature map when the input feature map's depth increases.
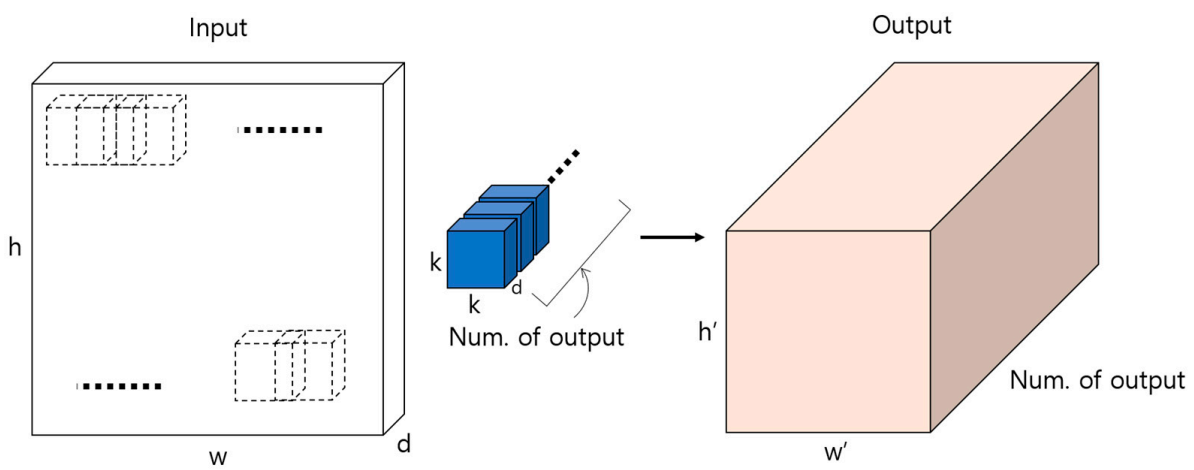

(a)

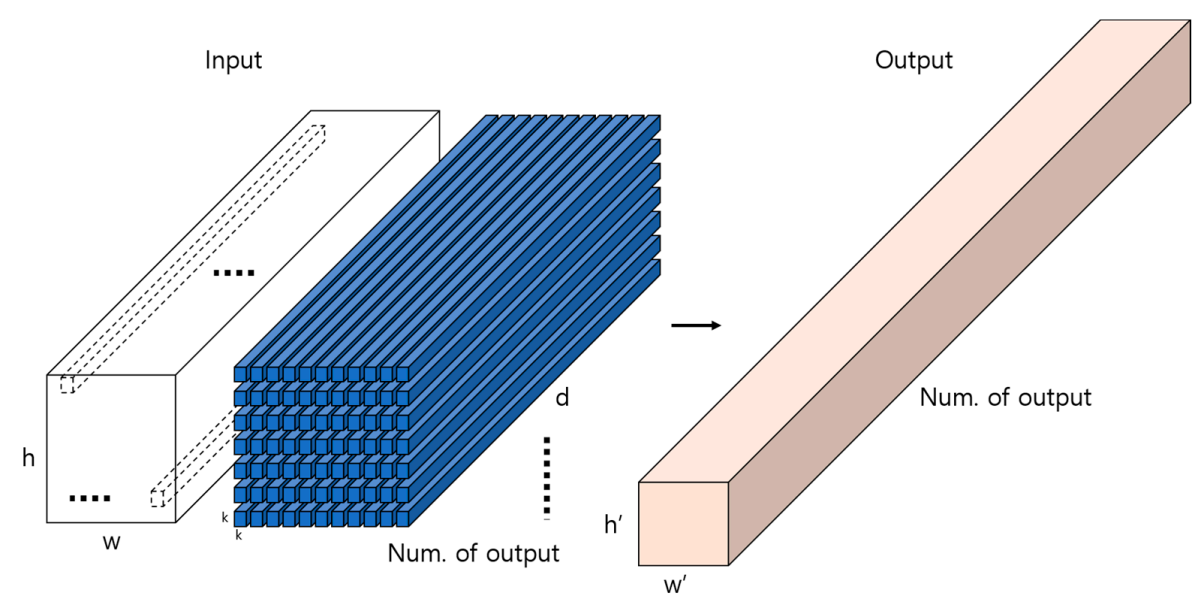

(b)

Figure 11. Example of obtaining output feature map via the convolutional layer. (a) Example of creating output feature map $\left(\mathrm{w}^{\prime} \times \mathrm{h}^{\prime} \times \mathrm{Num}\right.$. of output)) using as many as $\mathrm{k} \times \mathrm{k}$ filters of the same depth as the number of output on the input image $(\mathrm{w} \times \mathrm{h} \times \mathrm{d})$. (b) Example of convolution calculations and finding the output feature map when the input feature map's depth increases afterward. 
Based on Figure 11, this section analyzes the feature maps extracted from each layer of deep ResNet for the input ocular images as shown in Figure 12. As described in Figure 11, the output feature map's depth increased as the layers became deeper. Because this is difficult to express in 3D form, the feature maps for each depth are shown in the order from the top left to the bottom right, as seen in Figure 12. Figure 12a shows the feature map from Conv1 of Table 2. Figure 12b-d show the feature maps from the first, second, and third iterations of residual blocks in Conv2 of Table 2, respectively. Further, Figure 12e-g show the feature maps from the last residual blocks in Conv3, Conv4, and Conv5 of Table 2, respectively. For example, the feature maps obtained from Table 2's Conv1 have a size of $112 \times 112 \times 64$; therefore, 64 feature maps of size $112 \times 112$ are shown in the order from the top left to the bottom right, in Figure 12a.

As shown in Figure 12, feature maps are extracted from deeper convolutional layers, and as more abstract features are extracted, the area of the dominant feature in a feature map tends to grow larger. For example, in Figure 12a, features exist that show the original ocular shape and high-frequency edge components; however, in Figure $12 \mathrm{~g}$, the original ocular shape has disappeared, and the feature map consists of abstracted low-frequency features. Further, in Figure $12 b-d$, more convolution layers are performed than that in Figure 12a, and the ocular shape in the feature map disappears further. However, owing to the structure that preserves the original feature map before the convolution operation passes through residual blocks, the original ocular shape does not disappear entirely, and it can be seen that it is preserved to some degree. In Figure $12 \mathrm{f}, \mathrm{g}$, it can be seen that convolution layers have become even deeper, and the original ocular shape is almost not preserved. Further, it can be seen that the characteristics of low-frequency features, which are large and abstract in the feature map of Figure 12g, compensate to some degree for the reduction in recognition performance caused by misalignment between the enrolled and recognition images owing to the rough detection of the ocular area proposed in this study. Figure 12h shows 3-dimensional feature map image that is obtained by averaging all the feature map values of Figure $12 \mathrm{~g}$ in the channel (depth) direction, as well as the original ocular images. As shown in this figure, the magnitudes of feature map values are also large in the rough periocular region, which can prove that important features can be extracted from whole ocular region instead of only iris area.

Most of conventional studies on ocular recognition requires the procedure of iris segmentation $[8,13,14,16,17,22-27,35]$, as shown in Table 1 . This procedure includes the accurate detection of the boundaries and centers of pupil and iris, which takes much processing time and its accuracies are affected by the image quality and noises. Although there are previous studies without iris segmentation $[18,20,21]$ as shown in Table 1 , the recognition accuracies of their methods are low. Therefore, we propose ocular recognition based on rough pupil detection which does not include the procedure of accurate iris segmentation.

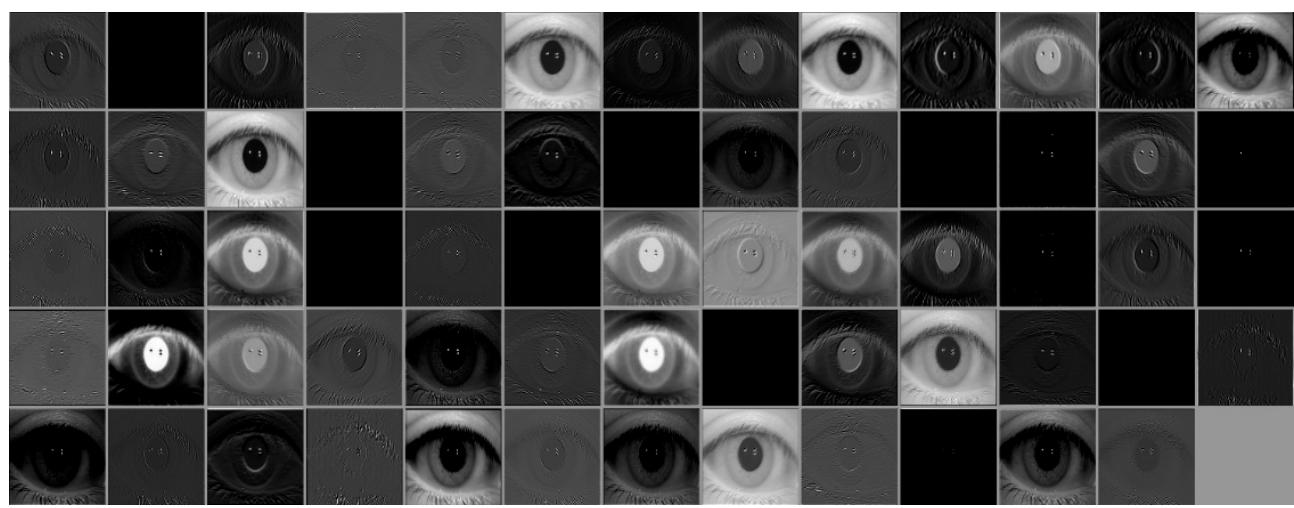

(a)

Figure 12. Cont. 


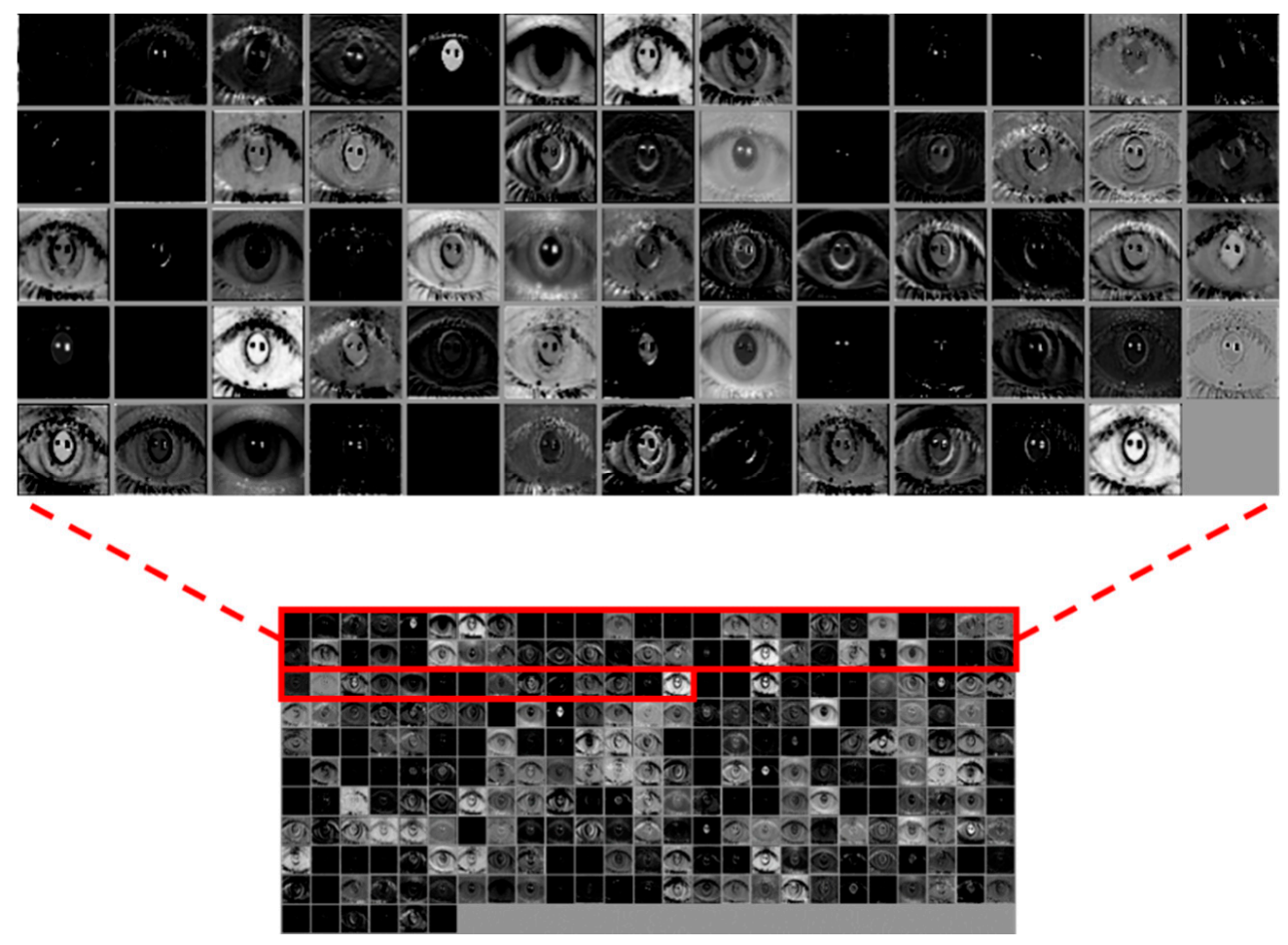

(b)

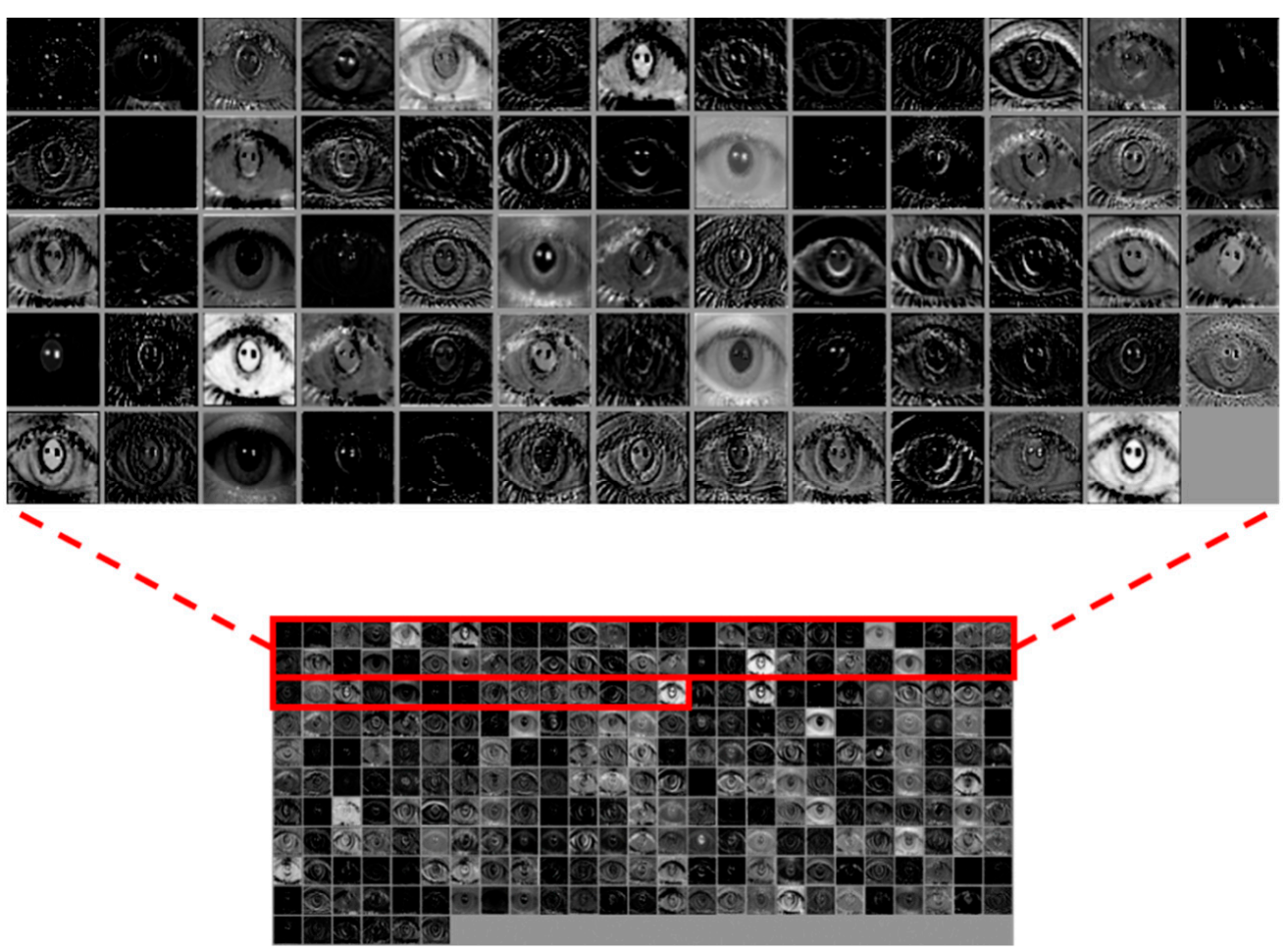

(c)

Figure 12. Cont. 


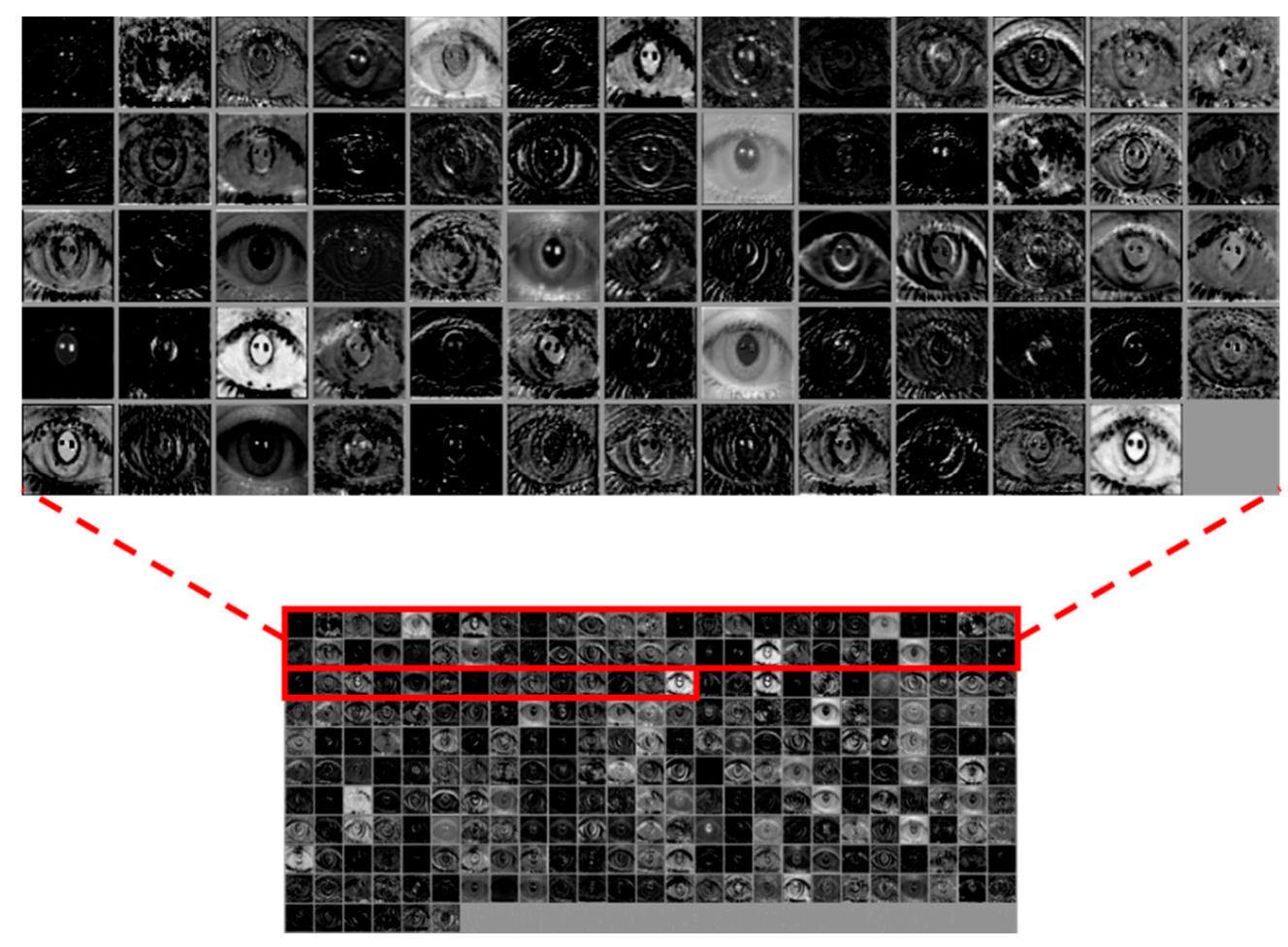

(d)

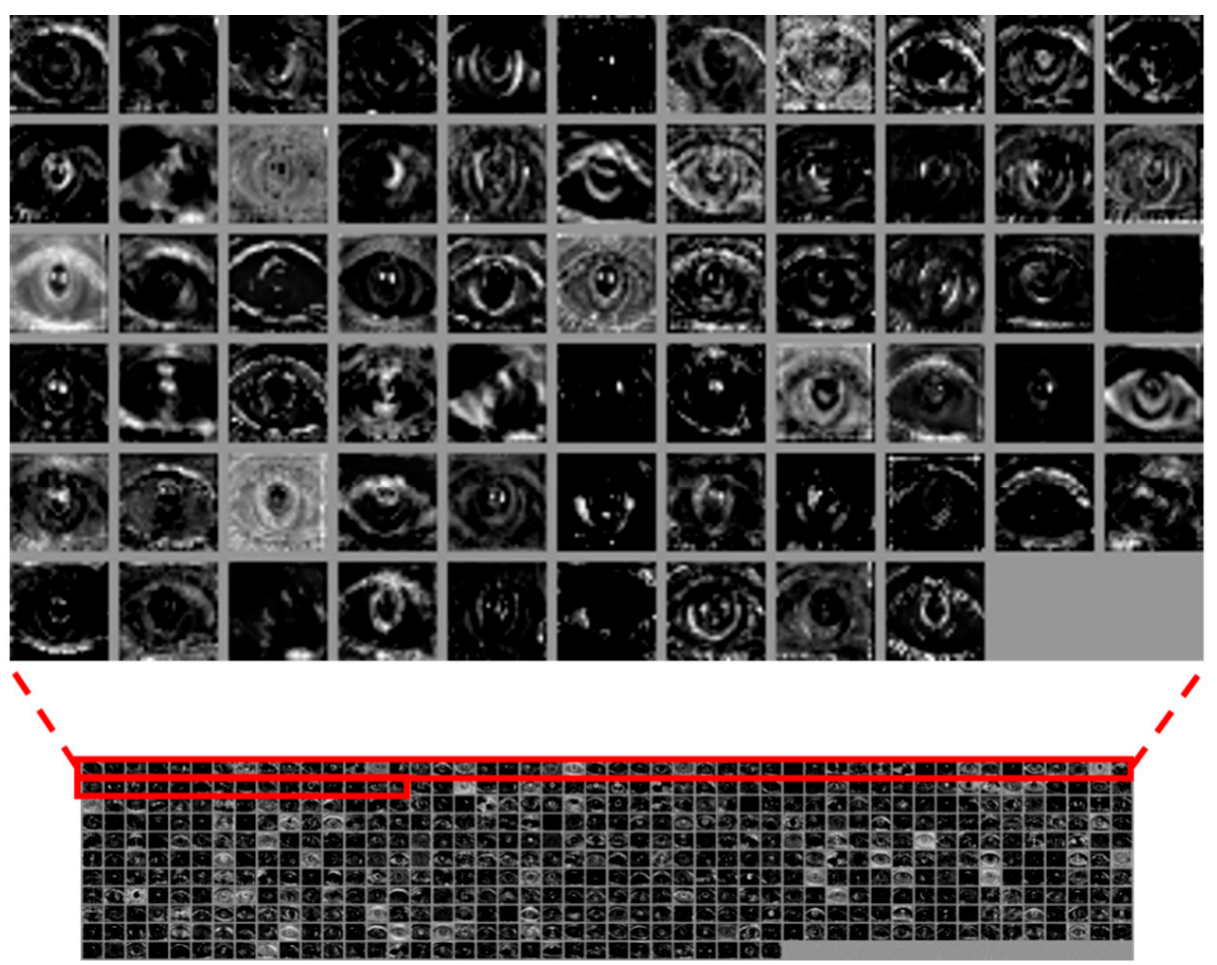

(e)

Figure 12. Cont. 


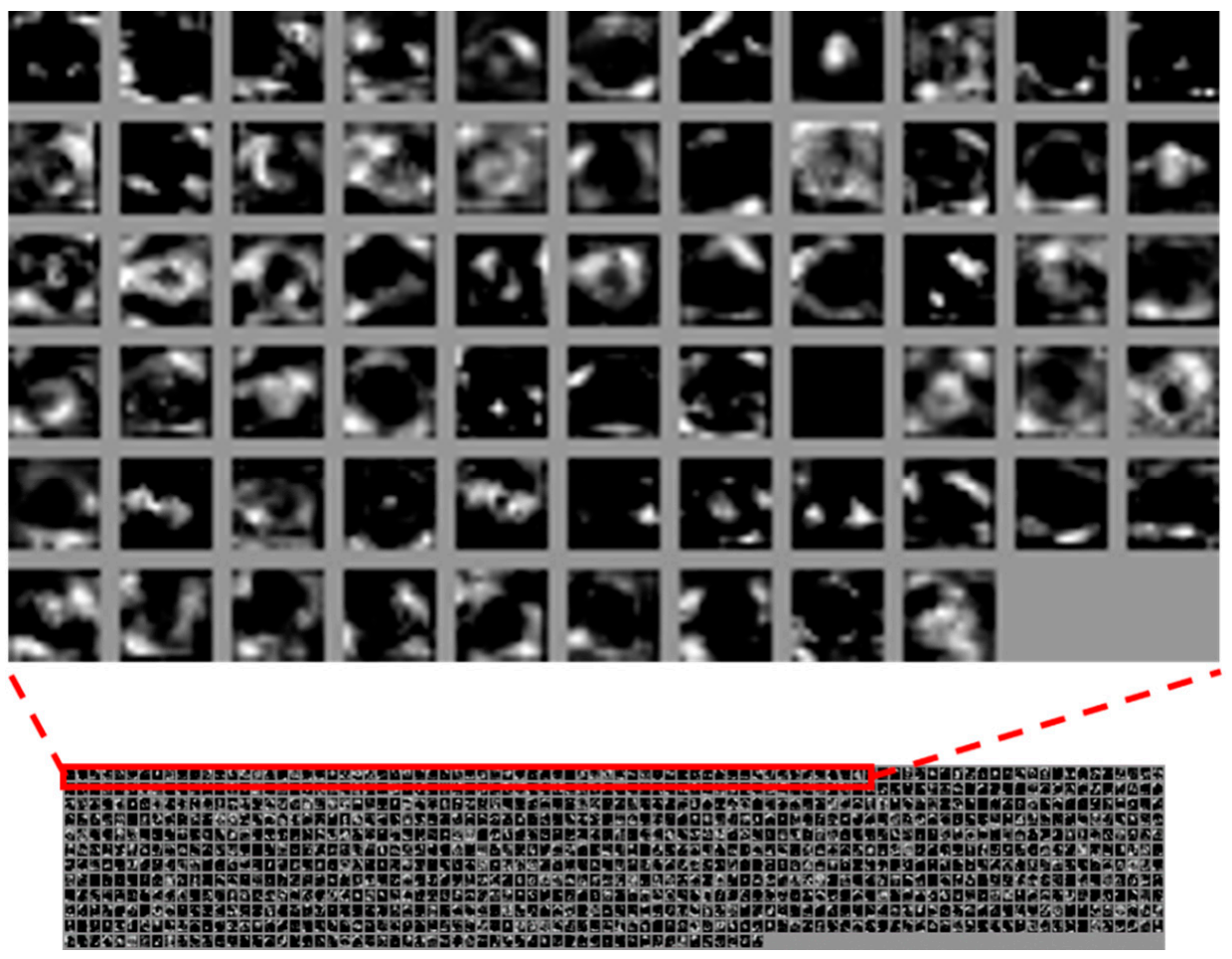

(f)

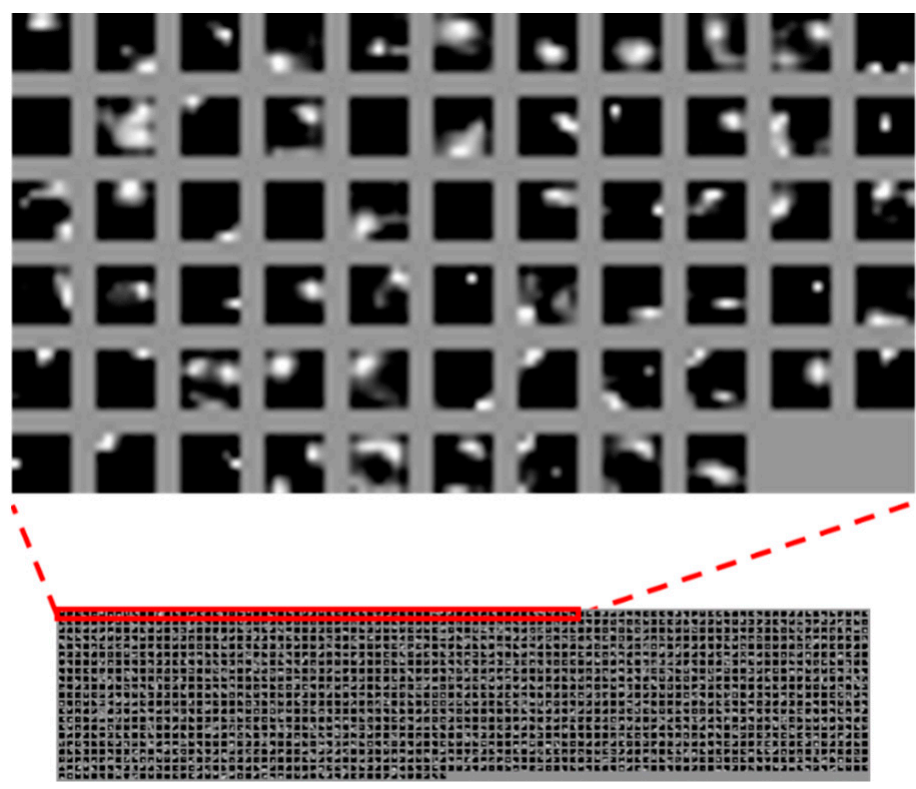

(g)

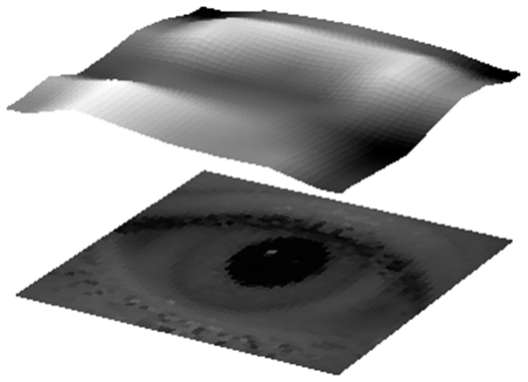

(h)

Figure 12. Examples of features maps extracted from each layer for the input ocular images. Feature maps from (a) Conv1 of Table 2, (b-d) the first, second, and third iterations of residual blocks in Conv2 of Table 2, respectively, and (e-g) the last residual blocks in Conv3, Conv4, and Conv5 of Table 2, respectively. (h) 3-dimensional feature map image that is obtained by averaging all the feature map values of $(\mathbf{g})$.

In our method, the rough pupil detection is performed by sub-block-based template matching as shown in Figures 2 and 3. As shown in these figures, the boundaries and centers of pupil and iris are not detected. Instead, the rough box position including pupil is located. Experimental results 
show that the positional difference between pupil centers in enrolled and recognized images by our sub-block-based template matching is about 7 10 pixels in $X$ and $Y$ axes, which are much larger than that by conventional iris segmentation method (in most cases, the difference is less than 1 2 pixels). However, the processing speed by our sub-block-based template matching is much faster than that by conventional iris segmentation $[6,65]$ as shown in Table 14. In addition, deep learning-based method is used in our research, and it could resolve the problem of reduced recognition accuracies due to the large difference between pupil centers in enrolled and recognized images by our sub-block-based template matching. Our deep learning-based method could also compensate the intra-class variation caused by openness of eye, in-plane rotation, off-angle, and specular reflections on glasses surface as shown in Figure 9.

Although the further extension of area, e.g., by the nose can produce the better accuracy, this kind of method cannot be used for the case that the nose area is occluded by wearing mask and its performance can be affected by the existence of mustache.

The accuracy by iris recognition can be better than that by our ocular recognition in case that the quality and image resolution of iris image are good. However, in case that the quality and image resolution are not good, the accuracy by our method using both iris and periocular regions is better than that only by iris or periocular recognition. This was proved as shown in Table 10. Our method can be difficult to be used in safety critical areas which requires high recognition accuracy. However, we can expect that our ocular recognition can be used for more reliable identification of people than iris or face recognition in case that the quality and image resolution of iris image are not good when the image is captured by the moving and uncooperative people at a distance with image blurring or other facial components including nose or mouth are not visible by wearing masks.

\section{Conclusions}

This study proposed the NIR camera-based ocular recognition method that uses deep features without any iris segmentation. The method uses a roughly identified eye area for recognition, without going through a process for detecting the pupil and iris boundary, the eyelid, and eyelashes, as this process requires a long processing time and its performance is affected by the image quality. To prevent decrease in recognition performance due to the roughly identified eye area, an ocular area somewhat larger than the iris area is used to perform recognition. Further, the method uses deep ResNet to resolve the problem of misalignment between the enrolled and recognition images, which can occur because of the recognition based on a roughly detected eye area, as well as the problem of reduced recognition performance caused by this misalignment. The proposed method analyzes the feature maps from various convolutional layers of the deep ResNet in order to examine the features that are important for ocular recognition in each of the layers. The results of tests using three open databases showed that the method proposed in this study has higher recognition accuracy than that of existing methods. However, it was found that FR cases occurred when severe in-plane rotation and off-angle happen, or glasses frame is included in the image. In addition, FA cases happened when the enrolled and recognition images were similar.

To resolve these problem, we plan to conduct a study in the future on a multimodal biometrics method that improves recognition performance by combining recognition information from both eyes. Further, we plan to study a method that can enlarge the CNN model's receptive field and extract features in order to resolve the problem of reduced recognition performance caused by the misalignment between the enrolled and the recognition images owing to the rough detection of the ocular region. In addition, we would plan to collect twin's iris dataset and have experiments with this self-collected dataset as future work.

Author Contributions: Y.W.L. and K.R.P. implemented the overall CNN system, and wrote this paper. K.W.K., T.M.H., and M.A. helped the experiments and analyzed results.

Funding: The research received no extra funding. 
Acknowledgments: This research was supported by the Basic Science Research Program through the National Research Foundation of Korea (NRF) funded by the Ministry of Education (NRF-2018R1D1A1B07041921), by the Bio \& Medical Technology Development Program of the NRF funded by the Korean government, MSIT (NRF-2016M3A9E1915855), and by the Basic Science Research Program through the National Research Foundation of Korea (NRF) funded by the Ministry of Education (NRF-2017R1D1A1B03028417).

Conflicts of Interest: The authors declare no conflict of interest.

\section{References}

1. Iris Recognition by Samsung Galaxy S8. Available online: http://www.samsung.com/global/galaxy/ galaxy-s8/security/ (accessed on 11 October 2018).

2. Flom, L.; Safir, A. Iris Recognition System. U.S. Patent US4641349, 3 February 1987.

3. Daugman, J.G. Biometric Personal Identification System Based on IRIS Analysis. U.S. Patent US5291560, 1 March 1994.

4. Daugman, J.G. High confidence visual recognition of persons by a test of statistical independence. IEEE Trans. Pattern Anal. Mach. Intell. 1993, 15, 1148-1161. [CrossRef]

5. Daugman, J.G. The importance of being random: Statistical principles of iris recognition. Pattern Recognit. 2003, 36, 279-291. [CrossRef]

6. Daugman, J.G. How iris recognition works. IEEE Trans. Circuits Syst. Video Technol. 2004, 14, 21-30. [CrossRef]

7. Shin, K.Y.; Nam, G.P.; Jeong, D.S.; Cho, D.H.; Kang, B.J.; Park, K.R.; Kim, J. New iris recognition method for noisy iris images. Pattern Recognit. Lett. 2012, 33, 991-999. [CrossRef]

8. Shin, K.Y.; Kim, Y.G.; Park, K.R. Enhanced iris recognition method based on multi-unit iris images. Opt. Eng. 2013, 52, 047201. [CrossRef]

9. Connaughton, R.; Sgroi, A.; Bowyer, K.; Flynn, P.J. A multialgorithm analysis of three iris biometric sensors. IEEE Trans. Inf. Forensic Secur. 2012, 7, 919-931. [CrossRef]

10. Daugman, J.G. New methods in iris recognition. IEEE Trans. Syst. Man Cybern. Part B Cybern. 2007, 37, 1167-1175. [CrossRef]

11. Monro, D.M.; Rakshit, S.; Zhang, D. DCT-based iris recognition. IEEE Trans. Pattern Anal. Mach. Intell. 2007, 29, 586-595. [CrossRef]

12. Park, H.-A.; Park, K.R. Iris recognition based on score level fusion by using SVM. Pattern Recognit. Lett. 2007, 28, 2019-2028. [CrossRef]

13. Li, P.; Ma, H. Iris recognition in non-ideal imaging conditions. Pattern Recognit. Lett. 2012, 33, $1012-1018$. [CrossRef]

14. De Marsico, M.; Nappi, M.; Riccio, D. Noisy iris recognition integrated scheme. Pattern Recognit. Lett. 2012, 33, 1006-1011. [CrossRef]

15. Păvăloi, I.; Ignat, A. Iris recognition using statistics on pixel position. In Proceedings of the IEEE International Conference on E-Health and Bioengineering, Sinaia, Romania, 22-24 June 2017; pp. 422-425.

16. Tan, T.; Zhang, X.; Sun, Z.; Zhang, H. Noisy iris image matching by using multiple cues. Pattern Recognit. Lett. 2012, 33, 970-977. [CrossRef]

17. Park, U.; Ross, A.; Jain, A.K. Periocular biometrics in the visible spectrum: A feasibility study. In Proceedings of the 3rd IEEE International Conference on Biometrics: Theory, Applications, and Systems, Washington, DC, USA, 28-30 September 2009; pp. 1-6.

18. Ross, A.; Jillela, R.; Smereka, J.M.; Boddeti, V.N.; Kumar, B.V.K.V.; Barnard, R.; Hu, X.; Pauca, P.; Plemmons, R. Matching highly non-ideal ocular images: An information fusion approach. In Proceedings of the 5th IAPR International Conference on Biometrics, New Delhi, India, 29 March-1 April 2012; pp. 446-453.

19. Rattani, A.; Derakhshani, R. Ocular biometrics in the visible spectrum: A survey. Image Vis. Comput. 2017, 59, 1-16. [CrossRef]

20. Cho, S.R.; Nam, G.P.; Shin, K.Y.; Nguyen, D.T.; Pham, T.D.; Lee, E.C.; Park, K.R. Periocular-based biometrics robust to eye rotation based on polar coordinates. Multimed. Tools Appl. 2017, 76, 11177-11197. [CrossRef]

21. Rodriguez, A.; Panza, J.; Kumar, B.V.K.V. Segmentation-free ocular detection and recognition. In Proceedings of the SPIE Defense, Security, and Sensing, Orlando, FL, USA, 25-29 April 2011; p. 80291W. 
22. Boddeti, V.N.; Smereka, J.M.; Kumar, B.V.K.V. A comparative evaluation of iris and ocular recognition methods on challenging ocular images. In Proceedings of the IEEE International Joint Conference on Biometrics, Washington, DC, USA, 11-13 October 2011; pp. 1-8.

23. Verma, S.; Mittal, P.; Vatsa, M.; Singh, R. At-a-distance person recognition via combining ocular features. In Proceedings of the IEEE International Conference on Image Processing, Phoenix, AZ, USA, 25-28 September 2016; pp. 3131-3135.

24. Oishi, S.; Ichino, M.; Yoshiura, H. Fusion of iris and periocular user authentication by adaboost for mobile devices. In Proceedings of the IEEE International Conference on Consumer Electronics, Las Vegas, NV, USA, 9-12 January 2015; pp. 428-429.

25. Tan, C.-W.; Kumar, A. Towards online iris and periocular recognition under relaxed imaging constraints. IEEE Trans. Image Process. 2013, 22, 3751-3765.

26. Lee, M.B.; Hong, H.G.; Park, K.R. Noisy ocular recognition based on three convolutional neural networks. Sensors 2017, 17, 2933.

27. Ahuja, K.; Islam, R.; Barbhuiya, F.A.; Dey, K. Convolutional neural networks for ocular smartphone-based biometrics. Pattern Recognit. Lett. 2017, 91, 17-26. [CrossRef]

28. Amos, B.; Ludwiczuk, B.; Satyanarayanan, M. Openface: A General-Purpose Face Recognition Library with Mobile Applications; CMU-CS-16-118; School of Computer Science, Carnegie Mellon University: Pittsburgh, PA, USA, June 2016; pp. 1-20.

29. Ribeiro, E.; Uhl, A.; Alonso-Fernandez, F. Iris super-resolution using CNNs: Is photorealism important to iris recognition? IET Biom. 2019, 8, 69-78. [CrossRef]

30. Zhao, Z.; Kumar, A. Improving periocular recognition by explicit attention to critical regions in deep neural network. IEEE Trans. Inf. Forensic Secur. 2018, 13, 2937-2952. [CrossRef]

31. Drozdowski, P.; Struck, F.; Rathgeb, C.; Busch, C. Detection of glasses in near-infrared ocular images. In Proceedings of the International Conference on Biometrics, Gold Coast, Australia, 20-23 February 2018; pp. 202-208.

32. Mahmood, A.; Uzair, M.; Al-Maadeed, S. Multi-order statistical descriptors for real-time face recognition and object classification. IEEE Access 2018, 6, 12993-13004. [CrossRef]

33. Reddy, N.; Noor, D.F.; Li, Z.; Derakhshani, R. Multi-frame super resolution for ocular biometrics. In Proceedings of the IEEE Conference on Computer Vision and Pattern Recognition Workshops, Salt Lake City, UT, USA, 18-22 June 2018; pp. 566-574.

34. Ahonen, T.; Hadid, A.; Pietikainen, M. Face description with local binary patterns: Application to face recognition. IEEE Trans. Pattern Anal. Mach. Intell. 2006, 28, 2037-2041. [CrossRef] [PubMed]

35. Sharifi, O.; Eskandari, M. Optimal face-iris multimodal fusion scheme. Symmetry 2016, 8, 48. [CrossRef]

36. Nguyen, K.; Fookes, C.; Ross, A.; Sridharan, S. Iris recognition with off-the-shelf CNN features: A deep learning perspective. IEEE Access 2018, 6, 18848-18855. [CrossRef]

37. Liu, N.; Zhang, M.; Li, H.; Sun, Z.; Tan, T. DeepIris: Learning pairwise filter bank for heterogeneous iris verification. Pattern Recognit. Lett. 2016, 82, 154-161. [CrossRef]

38. Gangwar, A.; Joshi, A. DeepIrisNet: Deep iris representation with applications in iris recognition and cross-sensor iris recognition. In Proceedings of the IEEE International Conference on Image Processing, Phoenix, AZ, USA, 25-28 September 2016; pp. 2301-2305.

39. Dongguk CNN Model for NIR Ocular Recognition (DC4NO) with Algorithm. Available online: http: / /dm.dgu.edu/link.html (accessed on 27 November 2018).

40. Kim, B.-S.; Lee, H.; Kim, W.-Y. Rapid eye detection method for non-glasses type 3D display on portable devices. IEEE Trans. Consum. Electron. 2010, 56, 2498-2505. [CrossRef]

41. Viola, P.; Jones, M.J. Robust real-time face detection. Int. J. Comput. Vis. 2004, 57, 137-154. [CrossRef]

42. Krizhevsky, A.; Sutskever, I.; Hinton, G.E. Imagenet classification with deep convolutional neural networks. In Proceedings of the 25th International Conference on Neural Information Processing Systems, Lake Tahoe, NV, USA, 3-6 December 2012; pp. 1097-1105.

43. Simonyan, K.; Zisserman, A. Very deep convolutional networks for large-scale image recognition. In Proceedings of the 3rd International Conference on Learning Representations, San Diego, CA, USA, 7-9 May 2015; pp. 1-14. 
44. He, K.; Zhang, X.; Ren, S.; Sun, J. Deep residual learning for image recognition. In Proceedings of the IEEE Conference on Computer Vision and Pattern Recognition, Las Vegas, NV, USA, 26 June-1 July 2016; pp. 770-778.

45. Deng, J.; Dong, W.; Socher, R.; Li, L.-J.; Li, K.; Fei-Fei, L. ImageNet: A large-scale hierarchical image database. In Proceedings of the IEEE Conference on Computer Vision and Pattern Recognition, Miami, FL, USA, 20-25 June 2009; pp. 248-255.

46. Nair, V.; Hinton, G.E. Rectified linear units improve restricted Boltzmann machines. In Proceedings of the 27th International Conference on Machine Learning, Haifa, Israel, 21-24 June 2010; pp. 807-814.

47. Heaton, J. Artificial Intelligence for Humans; Deep Learning and Neural Networks; Heaton Research, Inc.: St. Louis, MO, USA, 2015; Volume 3.

48. Multinomial Logistic Loss. Available online: http://caffe.berkeleyvision.org/doxygen/classcaffe_1_ 1MultinomialLogisticLossLayer.html (accessed on 30 November 2018).

49. CASIA-Iris Version 4. Available online: http://www.cbsr.ia.ac.cn/china/Iris\%20Databases\%20CH.asp (accessed on 9 November 2018).

50. Bottou, L. Stochastic gradient descent tricks. In Neural Networks: Tricks of the Trade; Springer: Berlin, Germany, 2012; pp. 421-436.

51. NVIDIA GeForce GTX 1070. Available online: https://www.nvidia.com/en-us/geforce/products/10series/ geforce-gtx-1070/ (accessed on 27 November 2018).

52. Jia, Y.; Shelhamer, E.; Donahue, J.; Karayev, S.; Long, J.; Girshick, R.; Guadarrama, S.; Darrell, T. Caffe: Convolutional architecture for fast feature embedding. In Proceedings of the 22nd ACM International Conference on Multimedia, Orlando, FL, USA, 3-7 November 2014; pp. 675-678.

53. Sensitivity Index. Available online: https://en.wikipedia.org/wiki/Sensitivity_index (accessed on 27 November 2018).

54. Rattani, A.; Derakhshani, R. On fine-tuning convolutional neural networks for smartphone based ocular recognition. In Proceedings of the IEEE International Joint Conference on Biometrics, Denver, CO, USA, 1-4 October 2017; pp. 762-767.

55. Shekar, B.H.; Bhat, S.S. Iris recognition using partial sum of second order Taylor series expansion. In Proceedings of the 10th Indian Conference on Computer Vision, Graphics and Image Processing, Guwahati, India, 18-22 December 2016; pp. 1-8.

56. Tan, C.-W.; Kumar, A. Accurate iris recognition at a distance using stabilized iris encoding and Zernike moments phase features. IEEE Trans. Image Process. 2014, 23, 3962-3974. [CrossRef]

57. Uhl, A.; Wild, P. Multi-stage visible wavelength and near infrared iris segmentation framework. In Proceedings of the 9th International Conference on Image Analysis and Recognition, Aveiro, Portugal, 25-27 June 2012; pp. 1-10.

58. Abdullah, M.A.M.; Dlay, S.S.; Woo, W.L. Fast and accurate method for complete iris segmentation with active contour and morphology. In Proceedings of the IEEE International Conference on Imaging Systems and Techniques, Santorini, Greece, 14-17 October 2014; pp. 123-128.

59. Proença, H.; Neves, J.C. IRINA: Iris recognition (even) in inaccurately segmented data. In Proceedings of the IEEE Conference on Computer Vision and Pattern Recognition, Honolulu, HI, USA, 21-26 July 2017; pp. 6747-6756.

60. Nigam, A.; Krishna, V.; Bendale, A.; Gupta, P. Iris recognition using block local binary patterns and relational measures. In Proceedings of the IEEE International Joint Conference on Biometrics, Clearwater, FL, USA, 29 September-2 October 2014; pp. 1-6.

61. Zhang, M.; Sun, Z.; Tan, T. Deformed iris recognition using bandpass geometric features and lowpass ordinal features. In Proceedings of the IEEE International Conference on Biometrics, Madrid, Spain, 4-7 June 2013; pp. 1-6.

62. Li, X.; Sun, Z.; Tan, T. Predict and improve iris recognition performance based on pairwise image quality assessment. In Proceedings of the IEEE International Conference on Biometrics, Madrid, Spain, 4-7 June 2013; pp. 1-6.

63. Zhang, Q.; Li, H.; Sun, Z.; Tan, T. Deep feature fusion for iris and periocular biometrics on mobile devices. IEEE Trans. Inf. Forensic Secur. 2018, 13, 2897-2912. [CrossRef] 
64. Wang, Z.; Li, C.; Shao, H.; Sun, J. Eye recognition with mixed convolutional and residual network (MiCoRe-Net). IEEE Access 2018, 6, 17905-17912. [CrossRef]

65. Arsalan, M.; Naqvi, R.A.; Kim, D.S.; Nguyen, P.H.; Owais, M.; Park, K.R. IrisDenseNet: Robust iris segmentation using densely connected fully convolutional networks in the images by visible light and near-infrared light camera sensors. Sensors 2018, 18, 1501. [CrossRef] 